\title{
Analyse situationnelle des programmes de santé de la reproduction
}

Population Council

Follow this and additional works at: https://knowledgecommons.popcouncil.org/departments_sbsr-rh How does access to this work benefit you? Let us know!

\section{Recommended Citation}

"Analyse situationnelle des programmes de santé de la reproduction." Population Council; Ministere de la Sante, Republique de Guinee; USAID, and BASICS, 1998. 


\section{MINISTÈRE DE LA SANTÉ PUBLIQUE}

\section{Analyse Situationnelle des Programmes de Santé de la Reproduction}

B) Basics

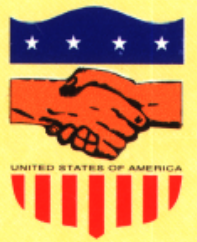

Population Council 


\section{PERSONNES AYANT PARTICIPE A L'ETUDE}

\section{Ministère de la Santé}

Responsable du projet : Dr Johanna Lucinda Austin, Directrice Nationale de la Santé Publique Chercheur principal : Dr Morissanda Kouyaté, Chef Division de la Santé de la Reproduction Responsable de la recherche : Dr Thierno Souleymane Diallo, Chef du SSEI

Assistante technique : Dr Soukeynatou Traoré, Chef de la section SMI/PF

\section{Consultants pour l'étude}

Coordonateur de l'étude : Mr Aliou Barry, Consultant, Stat View

Assistant de recherche : Mr Massé Camara, Consultant, CEFIB

Gestionnaire-Comptable : Mr Alpha Oumar Diallo, consultant, Stat View

\section{$\underline{\text { USAID }}$}

Mme Cathy Bowes, Chef de l'équipe Santé

Mr Peter Halpert, Adjoint chef de l'équipe Santé

Dr Mariama Ciré Bah, Chargée de programme

Elisabeth Kibour, Chargée de programme

\section{Population Council}

Dr Diouratié Sanogo, Directeur adjoint projet RO/AT II

Dr Nafissatou J. Diop, Chargée de programme

Dr Souleymane Kanon, Consultant

Dr Aristide Aplogan, Consultant

\section{$\underline{\text { BASICS }}$}

Dr Adama Koné, Directeur du Bureau Régional de BASICS

Dr Mutombo Wa Mutombo, Chargé de programme

Dr Mahamane Karki, Consultant

Mr Daniel Fassa Tolno, Consultant

Cette étude a été financée par la mission de l'USAID en Guinée. Elle a été réalisée par le Projet Recherches Opérationnelles et Assistance Tehnique (RO/AT II) en Afrique du Population Council. Le projet RO/AT est financé par la Division Population de l'USAID sour le contrat $\mathrm{N}^{\circ} \mathrm{CCP}-3030-\mathrm{C} 00-3008-00$, Stratégies pour Améliorer les Prestations de Services en matière de Planification Familiale. 


\section{REMERCIEMENTS}

Cette recherche opérationnelle est le fruit d'une parfaite collaboration multiinstitutionnelle dont les principaux acteurs sont le Ministère de la Santé, l'USAID, le Population Council et BASICS.

Le Ministère de la Santé remercie pour leur appui et leur contribution, les autorités politiques et administratives guinéennes à tous les niveaux et ses principaux partenaires que sont :

- L'USAID pour son soutien financier qui a permis la réalisation de cette Analyse Situationnelle.

- Le Bureau Régional du Population Council, Dakar, Sénégal, pour son assistance technique.

- Le Bureau Régional de BASICS, Dakar, Sénégal, pour sa contribution technique dans le volet « Survie de 1 enfant».

- Le FNUAP pour son appui logistique à travers le Bureau National du Recensement, Ministère du Plan et de la Coopération.

- L'ONG « Option Santé Familiale » (Population Services International) et l'UNICEF pour leur appui logistique dans le cadre de la supervision.

- L'Association Guinéenne pour le Bien Etre Familial (AGBEF), qui a bien voulu prêter ses locaux pour la saisie des données.

- La Stat-View Association pour l'assistance technique de son personnel.

Les remerciements vont particulièrement au Dr Nafissatou Diop, chargée de programme au Population Council dont l'expérience et l'expertise ont contribué à la réalisation de l'étude. Que le comité technique et les consultants $\mathrm{Mr}$ Aliou Barry, Mr Massé Camara, Mr Alpha Oumar Diallo, Dr Souleymane Kanon, Dr Aristide Aplogan, Dr Mutombo Wa Mutombo et Dr Mahamane Karki soient remerciés.

Aux enquêteurs qui, deux mois durant, ont contribué corps et âme à l'étude sur le terrain, et aux agents de saisie, sincères remerciements. 


\section{TABLE DES MATIERES}

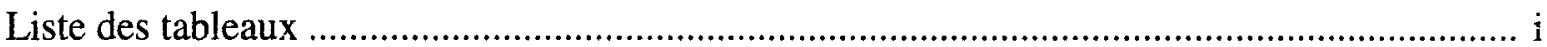

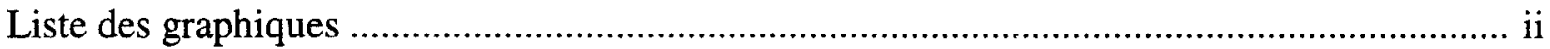

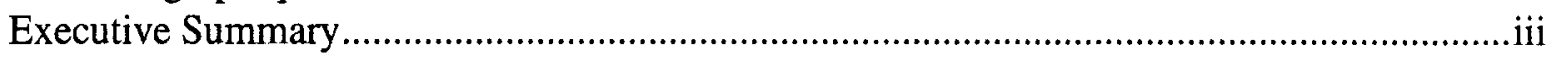

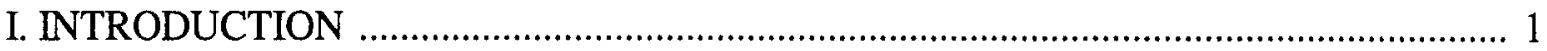

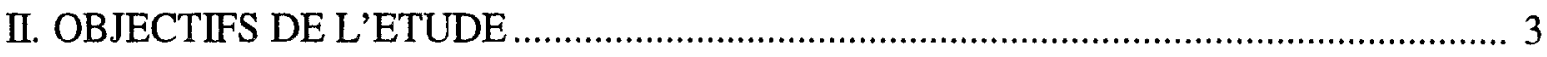

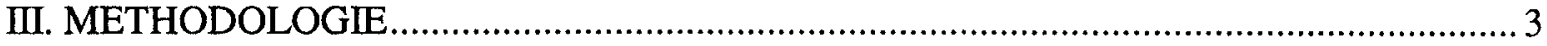

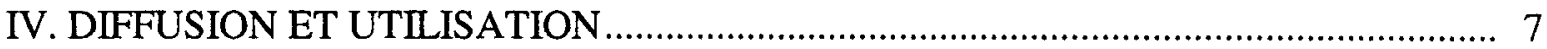

V. RESULTATS ET RECOMMANDATIONS ...................................................... 8

A. PRESENTATION GLOBALE DE L'ECHANTILLON ......................................... 8

B. ACCESSIBILITE AUX SERVICES DE SR/PF ..................................................... 12

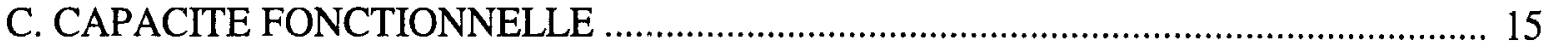

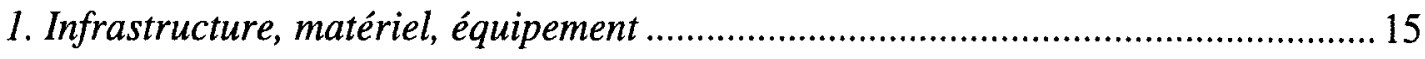

2. Expérience et formation des prestataires de service ......................................... 17

3. Activités et matériel IEC .................................................................................... 19

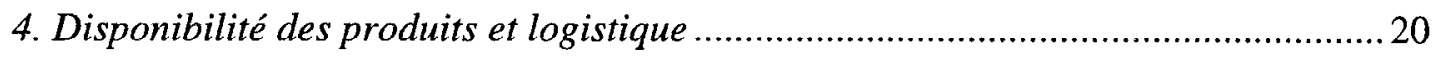

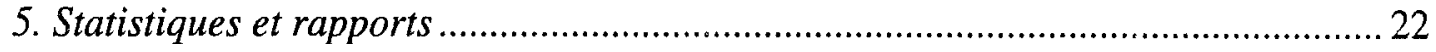

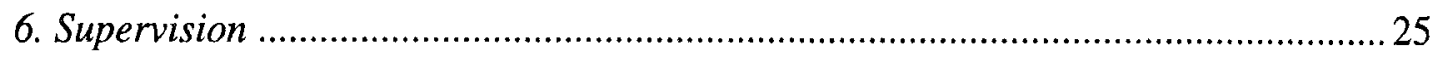

D. QUALITE DES SOINS DES FEMMES .............................................................. 27

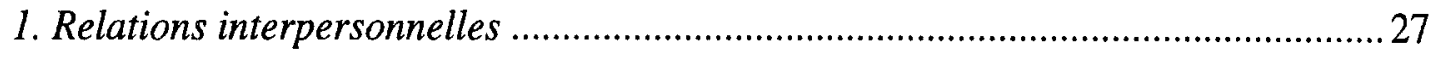

2. Choix des méthodes de PF ............................................................................. 28

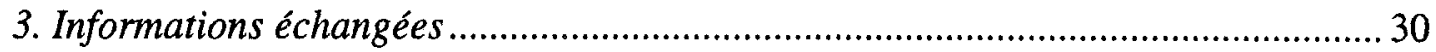

Concernant les visites prénatales ......................................................... 30

Concernant les consultations de MST/SIDA............................................... 31

Informations données aux clientes de $P F$.............................................. 31

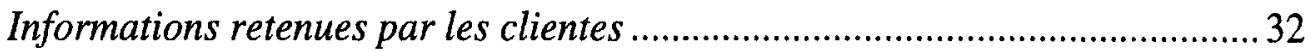

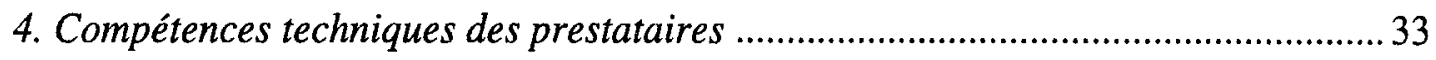

Procédures jugées nécessaires par les prestataires de $P F$............................ 33

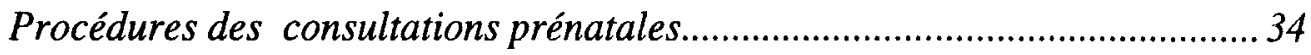

Aptitude des prestataires lors des consultations MST/SIDA ......................... 34

Procédures lors des consultations postnatales ........................................... 35 
5. Mécanismes pour encourager la continuité ……………....................................... 35

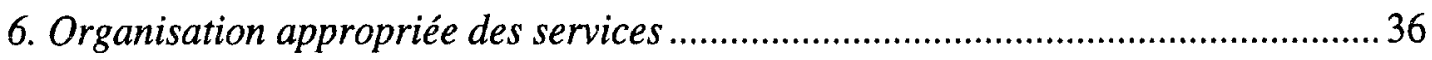

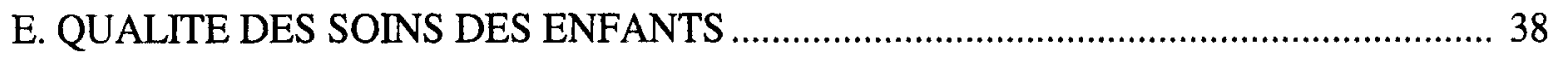

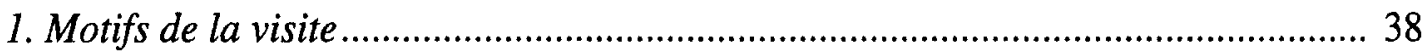

2. Evaluation de la maladie de l'enfant par le personnel...............................................40

3. Conseils et Communication interpersonnelle …………….........................................4 44

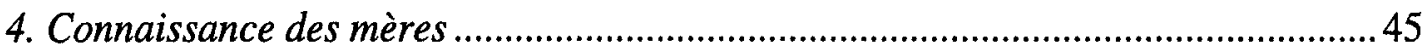

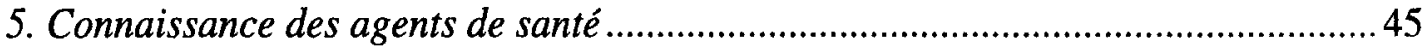

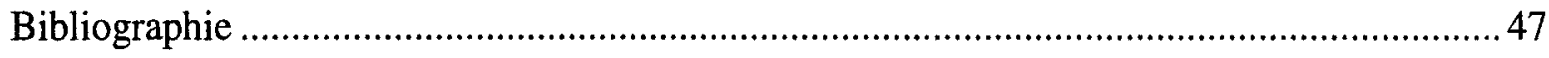




\section{LISTE DES TABLEAUX}

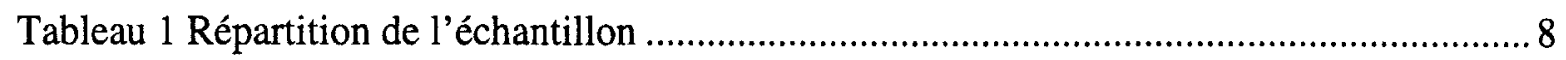

Tableau 2 Nombre d'observations SMI............................................................................. 9

Tableau 3 Indicateurs de fécondité des clientes PF et SMI ................................................... 10

Tableau 4 Caractéristiques socio-démographiques des prestataires .......................................... 11

Tableau 5 Disponibilité des services ............................................................................. 12

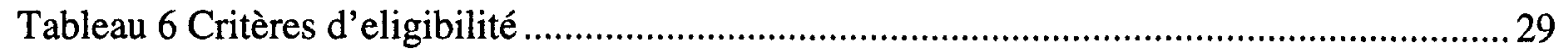

Tableau 7 Méthodes recommandées selon les objectifs de la cliente..................................... 30 


\section{LISTE DES GRAPHIQUES}

Graphique 1. Répartition des enfants malades selon le type de PPS ....................................... 9

Graphique 2. Caractéristiques des infrastructures ................................................................ 15

Graphique 3. Proportion des PPS disposant des matériels techniques .................................... 16

Graphique 4. Nombre moyen des prestataires titulaires selon le type de localité .................... 17

Graphique 5. Pourcentage des prestataires ayant reçu une formation .................................... 18

Graphique 6. Répartition des PPS selon le matériel IEC disponible ........................................ 19

Graphique 7. Répartition des PPS selon les médicaments disponibles .................................... 20

Graphique 8. Proportion des PPS où il existe des vaccins et médicaments périmés ................ 21

Graphique 9. Proportion de PPS ayant connu des ruptures .................................................. 21

Graphique 10. Proportion des PPS disposant des supports suivants pour le SNIS ..................22

Graphique 11. Répartition des PPS selon la disponibilité des statistiques PF ......................... 23

Graphique 12. Répartition des PPS selon la disponibilité des statistiques CPN .................... 24

Graphique 13. Répartition des PPS selon le nombre de supervisions reçus............................ 25

Graphique 14. Temps d'attente des clientes de PF ............................................................ 27

Graphique 15. Méthodes mentionnées avec les nouvelles clientes .........................................2 28

Graphique 16. Questions abordés lors de la consultation prénatale ......................................... 30

Graphique 17. Questions abordés lors de la consultation MST/SIDA ……..........................31

Graphique 18. Informations données aux clientes sur la noivelle méthode acceptée............. 31

Graphique 19. Connaissance de la contraception par les utilisatrices de pilule ........................32

Graphique 20. Procédure médicales lors des consultations PF ................................................. 33

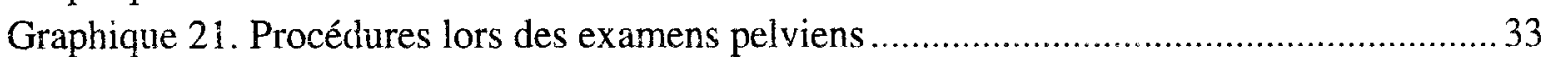

Graphique 22. Procédures lors des consultations prénatales .....................................................34

Graphique 23. Procédures lors des consultations MST/SIDA ……………………………........35

Graphique 24. Procédures lors des consultations postnatales ..................................................... 35

Graphique 25. Répartition d'enfants selon le nombre de motifs de consultation .................. 39

Graphique 26. Proportion d'enfants pour lesquels les symptômes ont été recherchés............ 40

Graphique 27. Proportion d'enfants selon les éléments dévaluation.......................................... 41

Graphique 28. Pourcentage d'enfants correctement évalués pour

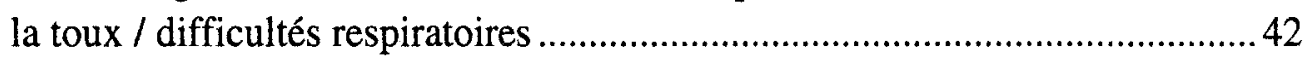

Graphique 29. Pourcentage d'enfants correctement évalués pour la diarrhée ...................... 42

Graphique 30. Pourcentage d'enfants correctement évalués pour la fièvre .......................... 43

Graphique 31. Pourcentage d'enfants dont l'état nutritionnel est évalué ............................... 44

Graphique 32. Pourcentage des mères correctement conseillées pour la prise en charge à domicile 


\section{EXECUTIVE SUMMARY}

In 1997 the Ministry of Health, in collaboration with USAID mission in Guinea, initiated a fiveyear program "Pour Renforcer les Interventions en Santé Reproductive et MST/SIDA" (PRISM). In order to better plan this new Reproductive Health (RH) program, and above all to measure its impact on key quality of service indicators, complete and reliable data on the various clinical subsystems was essential. This study serves to establish baseline data and provides information that will be useful for developing program activities.

The specific objectives are:

- Evaluate the quality and functional capacity of reproductive health services;

- Provide basic information for "PRISM " activity ;

- Provide quality of care and functional capacity indicators of the sub systems in order to evaluate the program;

- Identify specific interventions and operation research subject.

Nine data sources were used for the Guinea Situation Analysis. The data sources were:

-inventory of available facilities and services in the Service Delivery Points (SDPs);

-observation of the family planning service provider - client interaction;

-observation of the prenatal care provider - client interaction;

-observation of the postnatal care provider - client interaction;

-observation of the STD/AIDS care provider - client interaction;

-observation of medical consultations for sick children;

-interviews with Reproductive Health (RH) service-providers;

-exit interviews with clients who come for $\mathrm{MCH}$ care; and

-exit interviews with clients who come for FP services.

The study covered all of the SDPs offering FP services in Guinea. However, in order to have better data coverage at the national level and to take into account the other aspects of $\mathrm{RH}$, a number of non-FP clinics offering $\mathrm{MCH}$ services were included as well. All service-providers present the day of the visit were interviewed. All of the clients who came for FP care were interviewed as were a sample of prenatal, postnatal and STD/AIDs clients. A sample of children suffering from fever/malaria, cough/cold, diarrhea, acute respiratory infections or anemia/malnutrition was also observed, and the persons accompanying them were interviewed.

The study samples are:

Natural Regions + Conakry town

Number of prefectures

Number of SDPs

Number of FP observations

Number of prenatal observations

Number of postnatal observations 
Number of sick child observations

Number of FP interviews

Number of $\mathrm{MCH}$ interviews $\quad 1424$

Number of RH service-provider interviews $\quad 583$

The majority of the SDPs visited (83\%) are health centers. Other types of SDPs were also visited: the Donka University Hospital, Ignace Deen Hospital in Conakry, other hospitals (regional, prefecture-level and company hospitals), the AGBEF and Banankoro clinics. Most of these health care facilities (97\%) are public and are located in rural areas (59\%).

The remaining results are presented according to the following four criteria: accessibility, functional capacity, quality of care provided to women, and the quality of care provided to children.

\section{I- ACCESS TO RH/FP SERVICES}

Accessibility in terms of services provided. The majority of SDPs provide most of the services: FP, prenatal care, delivery, postnatal care, immunization, diarrheal case management, respiratory infection, malaria, and STDs/AIDS IEC. Aside from men and adolescent RH/FP services, HIV/AIDS tests, nutrition, post-abortion care, consultations for infertility, all of the services are generally available in more than $80 \%$ of the SDPs. Regarding IEC, one can see that FP IEC is more available than the others components.

Geographical accessibility. Based on the interviews with the MCH clients, the trip to the SDP takes an average of 47 minutes. The median trip time is 30 minutes whereas the maximum is 60 minutes.

Hours of operation: Approximately 29\% of the SDPs opened at the indicated time. Others began providing services after the established opening time, many from 30 to 60 minutes late. In $15 \%$ of the SDPs there was a delay of more than one hour. Such was also the case in $21 \%$ of the hospitals, $14 \%$ of the health centers and at the AGBEF clinics. Obviously, such opening delays limit access to health services, especially FP.

Cost of services: The MOH established an official fees for services. Based on interview data, the cost of services vary significantly depending on the type of SDP. An FP consultation costs 1000GF at AGBEF while in the public facilities it costs 5000GF. FP product prices also vary significantly depending on the type of SDP. As for the health centers, over-pricing for FP products exists especially in urban areas. The pill is sometimes sold for more than 5000GF in certain health centers, notably those in Conakry, Koundara, Mali, Beyla and Macenta. The average cost for services varies according to the type of consultation: The FP consultation costs an average of $1,148 \mathrm{GF}$, the $\mathrm{MCH}$ consultation costs 933 , and the sick child consultation costs 433 . The median $\mathrm{FP}, \mathrm{MCH}$ and sick child consultation costs are $1000 \mathrm{GF}, 600 \mathrm{GF}$ and $200 \mathrm{GF}$ respectively. 


\section{Recommendations:}

$>$ Efforts need to be made to extend the minimum package of services in all of the SDPs. Increased emphasis needs to be given to increasing the availability of adolescent and men's health services, nutritional services and post-abortion and sterility services.

$>$ There needs to be greater compliance with posted SDP hours of operation as well as increasing the public's awareness of extended hours for provision of services. This would help reduce the over-crowding that occurs in the morning hours.

The cost of consultations needs to be uniform, and official prices need to be posted in the SDPs.

\section{2- FUNCTIONAL CAPACITY}

Infrastructure. Waiting rooms exist in $91 \%$ of Guineas SDPs. Only $61 \%$ of the SDPs have sufficient seating. Functioning toilets or latrines exist in $85 \%$ of the SDPs but are not always accessible to clients. Water in sufficient quantity is generally not available in the SDPs. Out of 251 SDPSs visited, only 113 had enough water available for the SDP needs. The situation is more serious in rural areas where only $35 \%$ of the SDPs have enough water whereas the urban rate is $62 \%$. Electricity is even less available in rural areas (27\% of rural SDPs and $62 \%$ of urban SDPs). As for the consultations, one finds that in $91 \%$ of the SDPs there is an isolated room; and a $90 \%$ rate of visual and auditory privacy. Approximately $81 \%$ of the SDPs have clean facilities, and in 3 out of 4 SDPs the lighting is sufficient.

Equipment. In $30 \%$ of the SDPs there is no gynecological examination table. Only $84 \%$ of the SDPs have a tensiometer and closet. Nearly $27 \%$ of the SDPs lack gloves while $19 \%$ lack any size of speculum. We should remark that the study didn't observe the type of gloves. Among the SDPs offering IUD as a method, approximately $56 \%$ of the urban and $44 \%$ of the rural SDPs have no IUD kits. The norm calls for three different sizes of speculum to be available in the health centers. Only $13 \%$ of the SDPs have at least three speculum of each size.

Looking at equipment by category of SDP, it was found that the health centers are less likely to have a gynecological examination table (only $66 \%$ of the health centers), gloves, speculum and IUD kits. As for the FP materials, the AGBEF clinics seem to be the best supplied. Technical equipment for infants are lacking in almost all of the health centers in Guinea. In Mamou, for example, nearly nine out of ten do not have reflex hammers. In Labé as in Mamou 93\% of the SDPs lack a mucus extractor.

Service-providers. The data from the inventory questionnaire shows that $69 \%$ of the SDPs visited are not staffed with a tenured doctor. There are also fewer midwives, health technicians and nurses than there are Agents Techniques de Santé (ATS). Around one out of two SDPs do not have a midwife whereas only $2 \%$ of them lack an ATS. Between rural and urban areas there is considerable uneven distribution of personnel. Per SDP there are around five doctors in Conakry, three in other cities, and 0.2 in rural areas. The situation is almost the same for midwives.

The interview with the RH/FP service providers showed that the service-provider had worked on the average 6 years in the current SDP. The percentage of the RH/FP personnel offering FP services is $69 \%$. The personnel received their basic training in contraceptive technology and 
STD/AIDS took place an average of 12 years ago whereas other basic training took place an average of 16 years ago. This is normal when one considers that the average age of the serviceproviders is 41. However, many service-providers received in-service training notably in contraceptive technology and in prenatal consultations. One out of two service-providers received an in-service training 2-3 years ago.

IEC materials and activities. The inventory of IEC materials available in the SDPs shows that IEC materials are relatively rare (flip chart, audio-visual). In general the posters are used the most, whereas the other items are very rare or almost non-existent. By category of SDP activities, FP and STD/AIDS have the most IEC materials. Nearly four out of five SDPs have FP posters and more than $30 \%$ of them have STD/AIDS posters. However only $16 \%$ of the SDPs have prenatal consultation or postnatal posters. In $8 \%$ of the SDPs offering FP there is FP educational health talk. In $12 \%$ of the SDPs offering FP there are exteriors signs and in $37 \%$ of SDPs other internal visuals for FP.

Product Availability and Logistics. In general, on the day of the visit, contraceptive products were available in the majority of the SDPs offering FP. The pill was offered in $83 \%$ of the SDPs and was available in $97 \%$ of those offering the method. The other contraceptives such as spermicide, IUD and injectables were offered in $94 \%$ and $90 \%$ of the SDPs respectively. There is an written inventory of contraceptives in $88 \%$ of the SDPs offering FP. Of the 224 SDPs in Guinea offering FP, $85 \%$ claim to have a system for ordering new contraceptive supplies; $60 \%$ of these SDPs place their orders when their minimum safe stock level is reached whereas $4 \%$ of the SDPs wait until the stock runs out. In the six months leading up to the study, there had been serious stock-outs of nearly all of the products.

For the child survival products, it is mostly amoxycillin, quinine salts and Vitamin A that were lacking. The product inventory is most regularly done for childhood diseases ( $96 \%$ of the SDPs). $84 \%$ of the SDPs visited have an inventory of STD vaccines whereas for STD medicines the number is $81 \%$. In the six months leading up to the study, there had been serious stock-outs of nearly all of the products.

Information Systems. While the consultation registers are generally available, individual consultation records and child growth records are less available. The data available in the SDP registers show that statistical tracking of the FP activities is not done systematically in all of the SDPs. Out of 251 SDPs, only $40 \%$ have statistics available for a period of twelve consecutive months.

Supervision. Roughly 3 SDPs out of 10 collect information to be used in monitoring and evaluation. Most SDPs receive regular supervision. $92 \%$ of the SDPs had received at least one supervisory visit during the past three months. 


\section{Recommendations:}

$>$ The lack of water and electricity, especially in rural areas, needs to be addressed.

$>$ There needs to be a major effort to properly equip all health delivery structures, especially the health centers. The norms for standard equipment in all areas of $\mathrm{RH}$ are far from being respected.

$>$ It is necessary to recruit young qualified personnel. Periodic in-service training on new norms and procedures could also improve overall technical performance.

$>$ The IEC program for RH must be reinforced at the SDP level.

$>$ The frequent occurrence of stock-outs observed in the study shows that the system for ordering and supplying needs to be improved. The standardization of the system for placing orders, a revision of the method for determining the low-stock threshold alert, and logistics management training should be priorities.

$>$ While the percentage of SDPs receiving supervision is high, it appears from the number of stock-outs occurring, the poor record keeping, and the mediocre quality of care provided, that the content of the supervision needs to be reviewed and improved.

\section{3- QUALITY OF WOMEN'S HEALTH CARE}

Service-provider - client interaction. In general, the clients were received in a cordial manner (98\%) and were able to ask questions (94\% in FP). The highest median duration of the consultation was for new FP clients (15 minutes), whereas for the returning clients the median duration of consultation was 10 minutes.

Choice of method. The majority of the service-providers interviewed (more than 70\%) use a minimum age requirement to have access to the injectable, voluntary surgical contraception, the pill and the IUD. More than one out of three apply age limits for condoms and spermicides. It was found that there are service-providers who refuse to provide any contraceptive services to an unmarried woman or to a married woman without her husband's consent. In general the principal contraceptive methods proposed to a new client are the pill and the injectable.

Information provided to the clients. Directions for product use is the most common information provided to FP clients. What to do in case of problem with the method and the option to switch to another method dealt with less often. Exit interviews with FP clients show that they retain very little of the contraceptive method information given to them.

Among the first visit of prenatal clients, the most often discussed items are the client's age and reproductive history. The date of the most recent menstruation and medical history are rarely discussed.

It was found that only 59\% of the clients diagnosed with STD/AIDS were so informed. The other questions relating to the sexual behavior of the woman or of her partner are generally less often dealt with.

Technical competency of service-providers. During the pelvic examination for FP clients, conditions for asepsis are often not met. Further, sterile materials were very rarely used: $6 \%$ for examinations with speculum, and $28 \%$ for gloves. Very few clients were informed of the 
examination results. Not all of the service-providers are familiar with the accepted protocol for new client consultations and physical examinations. The quality of care provided to STD/AIDS clients is very poor. In less than half of the consultations did the service-provider look for mouth and skin lesions or check the lymph nodes. The quality of postnatal care provided to the clients was not very good.

Methods for encouraging continuity. Most of the new clients (55\%) who were not able to obtain their contraceptive products the day of the visit had to wait until their next menstruation. The other reasons were a stock-out (21\%) and the insertion of the IUD. The date of the next visit was given to nearly all of the FP and prenatal clients.

\section{Recommandations:}

$>$ Wide dissemination of $\mathrm{RH}$ norms and procedures to all levels of the health-care pyramid.

$>$ Provide formative supervision by assisting the health-care provider in dealing with clients.

$>$ During supervisory visits, highlight the importance of respecting hygiene norms to reduce the spread of infection.

$>$ Extend RH training to the ATS.

\section{4- QUALITY OF CARE GIVEN TO CHILDREN}

The median consultation duration for sick children was 8 minutes. A sick child could come for a consultation for several reasons. The most frequent reason for consultation was fever which was the case for $90 \%$ of the children in the sample. The results show that only $11 \%$ of the sick children had only one reason for the consultation. To adequately evaluate the child's illness, the serviceprovider generally must base the examination on three symptoms: coughing or respiratory difficulties, diarrhea and fever. Observation showed that in $61 \%$ of the cases the health care provider checked for all three symptoms.

Assessment of cough and respiratory difficulties. According to generally accepted norms and procedures, in order to correctly assess a child's cough or respiratory difficulties, one needs to measure respiratory frequency, listen to the chest, and check for a stridor or a whistling sound in the respiration. In $31 \%$ of the cases the service-providers undertook one of the three procedures. Only $18 \%$ of the children received all three of the established procedures.

Assessment of diarrhea. The following four procedures are necessary to assess diarrhea in a child: check for blood in stool, see if the child is thirsty and can drink, pinch the skin and check for sunken eyes. In only $9 \%$ of the cases did the service providers carry out all four procedures.

Assessment of fever. The health care provider should check to see if the child has a stiff neck, undress the child to see if there is a skin irritation and look for signs of ear infection. It is alarming that in only $6 \%$ of the cases were all of the 5 procedures accomplished. $24 \%$ of the children received none of the procedures.

Evaluation of the nutritional state of the child. The following four procedures were taken into account: undress the child to look for the weight, check for paleness of the palms, check for oedema 
of the feet, note the age/weight ratio. In $90 \%$ of the cases, the health care personnel failed to carry out all four of the procedures.

Evaluation of the child's eating patterns. In more than 7 out of 10 cases, no questions were asked in this area. One of the five planned questions were asked in $15 \%$ of the cases.

Evaluation of the vaccination history. In $62 \%$ of the cases the health worker did not ask for the vaccination record. It is no surprise to find that the vaccination status was assessed for only $49 \%$ of the sick children.

Caring for a sick child at home. The mothers almost never receive proper counsel on caring for a sick child at home. Only $0.3 \%$ of the mothers received all of the six generally accepted home care recommendations/instructions. The majority of mothers $(58 \%)$ received only one recommendation/instruction out of six. Unfortunately, they almost never check to see if the mothers understand the counsel given ( $97 \%$ of the cases).

The health worker - mother communication was assessed using three criteria. It is regrettable that in $79 \%$ of the cases, the mothers (or other accompanying persons) none of the three criteria were met. One of the three criteria was met for $19 \%$ of the mothers whereas in $9 \%$ of the cases two out of three criteria were met.

Mothers' knowledge of child health. In general, the mothers have a good understanding of the use of chloroquine. Almost all ( $99 \%$ ) know the correct dosage and the number of doses per day. However, the proportion of mothers who know the number of days that the chloroquine is to be administered is lower (77\%). Only $74 \%$ of the mothers interviewed were familiar with diarrheaprevention methods.

Health care workers' knowledge of child health. Fewer than $2 \%$ of the health workers know the dosage of Vitamin A for the treatment of measles. As for the groups targeted by the Programme Elargi de Vaccination, (PEV), there is also a lack of knowledge. Women in the 15 to 49 age group constitute the target group that is best known by the health workers (59\%).

\section{Recommendations:}

$>$ Take advantage of the opportunities identified by this study to accelerate the introduction of the PCIME Strategy (Prise en Charge Intégrée des Maladies de l'Enfant = Integrated Management of Childhood Illness) in the SDPs.

$>$ Encourage all health sector actors to support the development of an integrated action plan in favor of children.

$>$ Lobby donors in order to obtain the necessary means for reinforcing support to the Ministry of Health.

$>$ Improve the training of health personnel

$>$ Increase the number of persons qualified for taking charge of child survival.

$>$ Ensure the monitoring and evaluation of training.

$>$ Disseminate child survival norms and procedures widely. 


\section{5- DISSEMINATION AND USE OF RESULTS}

The data were disseminated and used as follows:

$>$ A two-day data interpretation workshop was organized in August for the presentation of the preliminary results to the technical committee extended to include the Ministry of the Plan and Cooperation and Africare.

$>$ A national-level workshop was held on November 9 and 10 bringing together nearly $140 \mathrm{MOH}$ officials from the central and regional levels, donors and NGOs. This workshop produced recommendations for dissemination of the findings and for improving the quality of health services.

$>$ A series of regional workshops are planned for early 1999 with a view toward involving fieldlevel service-providers.

$>$ The results of the Situation Analysis are currently serving as a basis for the development of nationnal health prọgräins süch às the "Programme Nationai de Déveioppement Sanitaire". (PNDS).

$>$ The data have permitted USAID and PRISM to review strategies and base-line indicators.

$>500$ copies of the final report were produced for widespread dissemination 


\section{I- INTRODUCTION}

\section{a) Contexte}

La Guinée couvre une superficie de $246000 \mathrm{Km}^{2}$. On distingue traditionnellement quatre régions naturelles qui diffèrent les unes des autres par le climat, le relief et les sols. Ce sont :

- la Basse Guinée, région ouest du pays, qui comprend les préfectures de Conakry (la capitale), Kindia, Coyah, Dubréka, Forecariah, Boffa, Fria, Boké et Télimélé ;

- la Moyenne Guinée ou zone du Fouta Djallon qui couvre un tiers environ du pays comprend les préfectures de Labé, Mamou, Dalaba, Pita, Lélouma, Gaoual, Koundara, Koubia, Mali et Tougué ;

- la Haute Guinée, région de savanes située à l'Est de la Guinée qui couvre sept préfectures : Kankan, Dabola, Kouroussa, Kérouané, Siguiri, Mandiana, Faranah et Dinguiraye ;

- la Guinée Forestière, région Sud du pays qui compte les préfectures de N'Zérékoré, Kissidougou, Guéckédou, Macenta, Beyla, Lola et Yomou.

La réorganisation du pays intervenue en 1994 a donné naissance à 7 régions administratives qui sont : Boké, Kankan, Faranah, N'Zérékoré, Kindia, Labé et Mamou. En plus, la ville de Conakry forme une entité administrative particulière. Le chef lieu de chaque région est la préfecture dont elle porte le nom.

Sur le plan démographique, la population guinéenne a été estimée à 7, 202, 957 millions d'habitants en 1996. Les femmes constituent $51 \%$ de la population. Environ $29 \%$ de cette population vit dans des villes de plus de 10000 habitants. L'indice synthétique de fécondité est de 5,7 enfants par femme et le taux d'accroissement naturel est de 2,8\%.

La planification familiale sous sa forme moderne est très récente en Guinée. Elle a été introduite pour la première fois en 1983 dans 3 sites de santé de Conakry: les CHU de Donka et de Ignace Deen et le centre de santé de Coronthie. Ce fut alors le point de départ des initiatives en faveur de la PF, telles que la création de cliniques modèles par l'AGBEF en 1984. En 1988, la République de Guinée a commencé à mettre en place un programme de soins de santé primaires visant en premier lieu la santé maternelle et infantile. Ce programme, en l'espace de quelques années, a fait des progrès remarquables en augmentant l'accessibilité et l'utilisation des services de protection de la mère et de l'enfant. La planification familiale a été introduite plus tardivement dans ce programme en 1991, mais a connu une évolution très rapide avec un accroissement constant de la disponibilité des services. En juin 1994, une politique de santé maternelle et infantile a été élaborée dont les principes s'inspirent de ceux de la politique nationale sanitaire et de la politique de la population de 1992. L'application de la politique sanitaire à travers le programme PEV/SSP/ME et le programme de SMI/PF a permis l'ouverture d'environ 335 centres de santé dans le pays. Cependant, plusieurs indicateurs de santé de la Guinée sont parmi les plus préoccupants du monde (USAID, Rapport d'Evaluation 1994). En effet, le taux de mortalité infantile est de 136 pour mille naissances. Le statut nutritionnel des femmes et des enfants apparaît comme étant très critique bien qu'aucune données fiables n'existe. Les maladies diarrhéiques sont les causes de $9 \%$ de décès d'enfants de moins de 1 an et représentent $15 \%$ des consultations médicales. Par contre le taux de couverture vaccinale a connu un accroissement important puisqu'il est passé de $3 \%$ en 1987 à $62 \%$ en 1996. Le taux de prévalance de 
séropositivité au VIH est estimé à environ 1,8\%. Le taux de mortalité maternelle (560 à Conakry et 800 dans les régions pour 100000 naissances) est parmi le plus élevé de la sous-région et constitue une préoccupation du gouvernement Guinéen. Et le taux de prévalence contraceptive reste bas avec $1 \%$ malgré la forte demande potentielle en services de planification familiale (EDS, 1992).

C'est dans ce cadre que la mission de l'USAID en Guinée a eu à financer en 1990 un programme d'intégration de la planification familiale dans les soins de santé primaire (FAMPOP) exécuté par PSI dans deux régions naturelles du pays. Pour une meilleure coordination, l'USAID travaille en étroite collaboration avec le FNUAP qui apporte son soutien financier aux deux autres régions. Outre les activités du secteur public, l'AGBEF une ONG nationale créée en 1984 et affiliée à l'IPPF, dispose aujourd'hui de cinq cliniques dans le pays.

La mise en oeuvre de la politique sanitaire s'effectue sur trois niveaux :

Au niveau central, on trouve les services centraux du Ministère de la Santé dont la Division de la Santé de la Reproduction. Il existe également deux Centres Hospitaliers Universitaires (CHU), structures de référence pour les soins spécialisés. Plusieurs autres centres spécialisés existent tels le Centre National de Formation et de Recherche en Santé, l'Institut de Nutrition et de Santé de l'Enfant, l'Institut National de Santé Publique, le Service de Santé Scolaire et Universitaire, le Service National de Santé des Travailleurs, le Service Statistiques Etudes et information et le Centre de transfusion sanguine.

Au niveau régional, on trouve un hôpital régional et une Inspection Régionale de la Santé (IRS) qui supervise des services médicaux. L'IRS assure également le suivi du Programme National de Soins de Santé Primaires et la Coordination des activités des ONG. Chaque région est découpée en préfectures et en sous-préfectures.

Au Niveau périphérique, on trouve la direction préfectorale de la santé (DPS), l'hôpital préfectoral, les centres de santé et les postes de santé. La DPS est responsable de la gestion des activités de base et l'hôpital préfectoral constitue le niveau de référence pour les centres de santé. Le centre de santé des sous-préfectures développe des programmes prioritaires (vaccinations, consultations prénatales, accouchements, planification familiale, etc..). Il apparaît que dans les 7 régions et à Conakry, presque tous les centres de santé $(84 \%)$ ont intégré la santé maternelle et infantile et une proportion importante a intégré la planification familiale (55\%) Les postes de santé sont au niveau des villages et des quartiers. Il existe aussi un programme pilote de Distribution à Base Communautaire (DBC) que le Ministère de la Santé teste à Kankan, N'Zérékoré et Faranah.

\section{b) Justification de l'étude}

Au courant de l'année 1997, le Ministère de la Santé a initié en collaboration avec la mission de l'USAID un programme quinquennal "Pour Renforcer les Interventions en Santé reproductive et MST/SIDA <<PRISM >>". L'objectif prioritaire de ce nouveau programme est d'accroître l'utilisation des services de planification familiale, de santé maternelle et infantile et de prévention des MST/SIDA à travers les 4 résultats intermédiaires suivants: 
1) Accroissement de l'accès à la PF/SMI ainsi que les services de prévention de MST/SIDA et les produits;

2) Amélioration de la qualité des soins de PF/SMI et celle des services de prévention MST/SIDA ainsi que les produits;

3) Accroissement de la demande et du comportement favorisant l'utilisation de la PF et de la SMI et les services de prévention de MST/SIDA, les produits et la pratique; et

4) Une meilleure collaboration entre les bailleurs de fonds, le Gouvernement, les organisations communautaires, les ONG et le secteur privé pour l'élaboration de stratégies efficaces.

Pour une meilleure planification de ce nouveau programme de Santé de la Reproduction et surtout pour mesurer son impact sur plusieurs indicateurs de la qualité des services, la disponibilité de données complètes et fiables sur les différents sous systèmes cliniques s'avère nécessaire. La présente étude a servi d'enquête pré-intervention pour évaluer la situation initiale et fournir des informations pouvant contribuer à formuler les interventions. Les résultats de l'étude peuvent aider à identifier des thèmes prioritaires de recherche opérationnelle. Finalement ce même type d'étude à la fin de l'intervention, permettra de mesurer les effets des activités des programmes de santé de la reproduction.

\section{OBJECTIFS DE L'ETUDE}

L'objectif à long terme de l'étude est d'apporter une assistance au Ministère de la Santé dans la planification de l'amélioration des services de santé de la reproduction.

Les objectifs immédiats sont au nombre de quatre:

- Evaluer la qualité et le fonctionnement des services de santé de la reproduction ;

- Fournir des informations de base aux activités de "PRISM ";

- Fournir des indicateurs de base de la qualité des soins et de la capacité de fonctionnement des sous systèmes afin de pouvoir évaluer les performances des programmes ;

- Identifier des actions pertinentes en matière de stratégies d'intervention et de recherche opérationnelle.

\section{METHODOLOGIE}

\section{a) Cadre conceptuel}

La bonne gestion des programmes de santé, qui constitue un défi pour les responsables africains, nécessite une connaissance suffisante des différentes composantes du système de prestation de services de santé qui sont : le personnel, les infrastructures et leur équipement, et l'offre des services. 
Le développement et l'évolution de la méthodologie des études d'Analyse Situationnelle vient du besoin des responsables de programmes de planification familiale d'obtenir des informations sur la disponibilité, l'accessibilité et la qualité des soins offerts par leurs programmes. D'une manière générale, une Analyse Situationnelle cherche à évaluer la capacité fonctionnelle qu'ont les structures de santé à offrir des services de qualité. C'est une étude diagnostique des forces et des faiblesses des composantes d'un programme de santé et de la qualité des soins, à partir d'un échantillon représentatif des PPS. Initialement conçue pour la planification familiale, elle a été adaptée au besoin des autres programmes de santé de la reproduction.

La première Analyse Situationnelle a été exécutée en 1989 au Kenya par le Population Council. Dès lors cette méthodologie a été améliorée, adaptée et utilisée dans plusieurs pays d'Afrique, d'Amérique Latine, d'Asie et du Moyen Orient. L'approche d'Analyse Situationnelle permet de réunir "rapidement" des informations sur les différentes composantes du programme et les problèmes qui lui sont liés.

\section{b) Echantillonnage}

Il existe trois unités de sondage dans l'étude : le Point de Prestation de Services (PPS), le personnel du PPS et les client(e)s venant au PPS. Neuf sources de données sont rassemblées dans le cadre de l'étude d'Analyse Situationnelle de la Guinée. Pour chaque type de données, des instruments ont été mis au point. L'information a été collectée à partir des sources suivantes :

- L'inventaire des installations disponibles et des services offerts dans les PPS ;

- L'observation de l'interaction entre la cliente et le prestataire de services de PF ;

- L'observation de l'interaction entre la cliente et le prestataire de soins prénatals ;

- L'observation de l'interaction entre la cliente et le prestataire de soins post natals ;

- L'observation de l'interaction entre la cliente et le prestataire de soins MST/SIDA ;

- L'observation de l'interaction entre l'enfant malade et le prestataire des soins pour la survie de l'enfant ;

- Les interviews avec les prestataires de services de SR ;

- Les interviews avec les clientes venues pour soins de santé maternelle et Infantile et ;

- Les interviews avec les clientes venues pour planification familiale.

L'étude a porté sur tous les PPS offrant des services de planification familiale fonctionnels sur le territoire guinéen. Cependant, pour avoir une bonne représentativité des données au plan national et pour prendre en compte les autres volets de la SR, un certain nombre de structures de santé non intégrées en PF mais offrant les autres services de SMI ont été rajoutés.

Tous les prestataires de service présents le jour de la visite ont été interviewés. Toutes les clientes venues pour des soins de planification familiale le jour de la visite ont fait l'objet d'une interview et un échantillon des clientes venues pour soins prénatals, postnatals et MST/SIDA ont été également interviewé. Un échantillonnage d'enfants malades avec des symptômes de fièvre, de paludisme, de toux et rhume, de diarrhée, d'infections respiratoires aiguës ou d'anémie/malnutrition a été également observé et leurs accompagnants interviewés. Dans les PPS à faible affluence, tous les enfants malades ont été observés. Par contre dans les PPS à grande affluence, un rythme d'observations des enfants malades a été défini. Pour le même motif de 
consultations, un enfant malade sur trois a été observé et sa mère interviewée à la sortie de la consultation.

\section{c) Activités de l'étude}

\section{Phase préparatoire}

La préparation de l'enquête s'est déroulée en plusieurs phases:

- Un comité technique a été crée en mars 1998 pour suivre l'évolution des activités de l'étude. Ce comité de 23 membres comprenait l'équipe technique de l'étude: le responsable de l'étude, le chercheur principal et son adjointe, l'assistant technique de Population Council et de Basics, et le coordonnateur. Le personnel des services ci-après a complété le comité:

- au Ministère de la Santé: la Division Santé de la Reproduction avec toutes ses sections, le Service Statistiques Etudes et Informations, le programme de lutte contre les maladies diarrhéiques, le Service de Nutrition et le programme de lutte contre le SIDA;

- les organisations internationales: USAID, FNUAP, OMS, UNICEF;

- les ONG et autres partenaires: AGBEF, PRISM et OSFAM/PSI

Le rôle de ce comité était de s'assurer de la qualité technique et scientifique de l'étude en:

- approuvant les principaux documents de l'étude tels que les questionnaires de collecte des données, le plan d'échantillonnage et le calendrier détaillé des activités, les rapports préliminaires et final de l'étude;

- assistant aux importantes réunions organisées dans le cadre de l'exécution des activités, le plan d'analyse et la définition des grands axes des recommandations;

- assurant le suivi de l'application des recommandations issues du séminaire de diffusion.

- La révision des questionnaires a été faite par les membres de ce comité du 9 au 13 Mars 1998. Il s'agissait d'adapter les questionnaires aux spécificités des programmes de santé de la Guinée.

- L'identification des sites de l'étude au niveau de toutes les régions administratives. Plusieurs mécanismes (messages à la radio du Département, communiqués à la radio nationale, lettres etc..), ont permis d'informer les IRS et les DPS de la tenue prochaine de l'enquête et de mettre à jour les listes des PPS fonctionnels offrant la PF dans chaque région. Ces informations ont permis d'élaborer un calendrier de tournée pour la collecte des données.

\section{Organisation de la collecte des données}

- La formation des équipes d'enquêtes. Elle s'est déroulée au mois d'Avril/Mai et a duré 15 jours. La formation a été assurée par 7 personnes des organismes suivants: Ministère de la Santé, Population Council et BASICS. Il s'agissait essentiellement d'apprendre à 32 personnes à se familiariser et à maîtriser les questionnaires et le protocole d'organisation de la collecte. Pendant cette formation, il y a eu des cours théoriques et pratiques sous forme de jeux de rôles. La formation en salle a été complétée par des pré-tests sur le terrain. 
- Le profil et les tâches du personnel de collecte. La collecte a été effectuée par 30 personnes réparties en 8 équipes. Chaque équipe était composée d'un superviseur (médecin), de deux agents de santé et d'une enquêtrice. Exceptionnellement, dans deux équipes qui couvraient les régions à faible intégration de PF, il n'y a eu qu'un seul agent de santé au lieu de deux.

Le superviseur était chargé de faire l'inventaire des installations et en cas d'affluence d'assister l'observatrice ou l'enquêtrice. Il a coordonné aussi le travail de l'équipe, informé les responsables locaux. Il était également chargé de la logistique et du bon déroulement du travail.

Les agents de santé ont assuré les observations des interactions entre les prestataires et les client(e)s/patients et les interviews des prestataires de service.

L'enquêtrice était responsable de l'interview des clientes PF et SMI à la sortie de la consuitation.

- La tournée de collecte. Elle a eu lieu du 19 mai au 15 juillet 1998 simultanément dans toutes les régions administratives de la Guinée. Les questionnaires ont été contrôlés à plusieurs niveaux: 1) sur le terrain tous les jours par toute l'équipe de collecte; 2) par le personnel d'encadrement chargé de la supervision; et 3) lors des réunions de mise en commun.

\section{Traitement des données}

Les données collectées dans le cadre de cette Analyse Situationnelle ont toutes été saisies sur micro-ordinateurs à l'aide du logiciel Epi-info. Pour la saisie des données, 9 programmes ont été élaborés. La saisie des données a été faite par 8 opérateurs de saisie experimentés. Elle a duré un mois et demi. Après la saisie, tous les fichiers ont été nettoyés avec des contrôles de cohérence.

\section{d) Limites de l'étude}

Dans l'Analyse Situationnelle de la Guinée des biais liés à la méthode de selection des unités de sondage existent. Mais des mesures avaient été prises à l'avance pour apporter quelques corrections.

Limites liées au choix des PPS. Dans l'approche utilisée, toutes les structures de santé intégrées en PF ont été incluses dans l'échantillon. I y a eu ensuite une sélection aléatoire des PPS n'offrant pas la PF en vue d'une meilleure représentativité des données. En effet, les régions de la Basse et de la Moyenne Guinée comptent moins de PPS intégrés en PF que les deux autres régions. Pour assurer à l'échantillon une bonne représentativité du point de vue de la SMI, les mesures suivantes avaient été prises:

- Augmentation de la taille initiale de l'échantillon de 227 à 251 PPS;

- L'inclusion de tous les PPS de Conakry dans l'échantillon;

Limites liées à la sélection des prestataires. En principe, tous les prestataires des services de santé de la reproduction qui travaillent dans les PPS visités devraient être inclus dans 
l'échantillon. Cependant, avec la méthodologie des études d'Analyse Situationnelle, seuls les prestataires présents le jour de l'enquête peuvent être inclus dans l'échantillon. Pour parer à toute exclusion de l'échantillon d'une importante proportion de prestataires de services de Santé de la Reproduction, tous les PPS de l'échantillon étaient avisés de la date de visite de l'équipe d'enquête.

Limites liées au choix des clientes et des enfants malades. Toutes les clientes venues au PPS le jour de l'enquête devraient faire l'objet d'observations au moment de la consultation et soumises à des interviews à la sortie. Cependant, comme les observations allaient beaucoup plus vite que les interviews, certaines clientes observées n'ont pu être interviewées. Pour ce qui concerne la survie de l'enfant, compte tenu de l'affluence dans certains PPS, une instruction ferme avait été donnée quand au rythme d'observations des enfants malades: pour le même symptôme, 1 enfant malade sur trois devait être observé et son accompagnant interviewé.

\section{DIFFUSION ET UTILISATION}

Les données ont ćté diffusées et utilisées comme suit.

- Un atelier d'interprétation des données de deux jours a été organisé en Août 1998 en vue de présenter les résultats préliminaires au comité technique, élargi aux représentants du Ministère du plan et de la coopération et à l'ONG Africare.

- Du 9 au 10 Novembre, un atelier national regroupant près de 140 responsables du Ministère de la Santé au niveau central et régional, les bailleurs de fonds et les ONG a été organisé à Conakry. Les participants ont eu à faire des recommandations qui ont été inclues dans le rapport.

- Une série d'ateliers de diffusion dans les régions ont été programmées pour le début de l'année 1999 en vue d'impliquer les prestataires de services de santé dans les structures périphériques.

- Les résultats de l'Analyse Situationnelle servent actuellement d'indicateurs de base pour l'élaboration de programmes nationaux de santé comme le Programme National de Développement Sanitaire (PNDS).

- Les données permettent aussi à la mission de l'USAID et à PRISM et d'autres agences de collaboration de rafiner leurs stratégies et d'avoir des indicateurs de base.

- Ce rapport final a été produit en 500 exemplaires pour une large distribution. 


\section{PRINCIPAUX RESULTATS ET RECOMMANDATIONS}

\section{A- PRESENTATION GLOBALE DE L'ECHANTILLON}

\section{1- Les PPS.}

Dans le cadre de l'Analyse Situationnelle de la Guinée, toutes les 4 régions naturelles ont été couvertes, ainsi que la capitale Conakry. Au total, 251 structures de santé ont été visitées dont la majorité se trouvent en Guinée Forestière (31\%).

A Conakry, la capitale, tous les Points de Prestations de Services (PPS) ont été couverts. Selon la région administrative, N'Zérékoré compte le plus grand nombre de PPS visités au cours de cette étude. Cette inégalité s'explique par la répartition géographique des PPS qui est très variable et surtout par l'intégration différentielle de la PF dans les PPS selon la région.

Tableau $N^{0} 1$ : Répartition de l'échantillon de l'étude

\begin{tabular}{|c|c|c|c|c|c|c|}
\hline $\begin{array}{l}\text { Région } \\
\text { Naturelle }\end{array}$ & $\left\{\begin{array}{l}\text { Nombre } \\
\text { de Préfectures }\end{array}\right.$ & $\begin{array}{l}\text { Nombre } \\
\text { de PPS }\end{array}$ & $\left\{\begin{array}{l}\text { Nombre } \\
\text { d'observations } \\
\text { PF }\end{array}\right.$ & $\begin{array}{l}\text { Nombre } \\
\text { d'interviews } \\
\text { PF }\end{array}$ & $\begin{array}{l}\text { Nombre } \\
\text { d'interviews } \\
\text { SMI }\end{array}$ & $\mid \begin{array}{l}\text { Nombre } \\
\text { D'interviews } \\
\text { De prestataires }\end{array}$ \\
\hline Conakry & 1 & 23 & 37 & 36 & 171 & 97 \\
\hline Basse Guinée & 8 & 42 & 23 & 23 & 229 & 98 \\
\hline Moyenne Guinée & 10 & 50 & 52 & 52 & 255 & 98 \\
\hline Haute Guinée & 8 & 58 & 64 & 36 & 350 & 114 \\
\hline Guinée Forestière & 7 & 78 & 131 & 79 & 419 & 176 \\
\hline TOTAL & \multirow[t]{2}{*}{34} & \multirow[t]{2}{*}{251} & \multirow[t]{2}{*}{307} & \multirow[t]{2}{*}{226} & \multirow[t]{2}{*}{1424} & \multirow[t]{2}{*}{583} \\
\hline Région Administr & & & & & & \\
\hline Conakry & 1 & 23 & 37 & 36 & 171 & 97 \\
\hline Boké & 5 & 24 & 9 & 9 & 117 & 45 \\
\hline Kindia & 5 & 26 & 18 & 18 & 151 & 66 \\
\hline Mamou & 3 & 15 & 7 & 7 & 78 & 27 \\
\hline Labé & 5 & 27 & 41 & 40 & 139 & 58 \\
\hline Faranah & 4 & 33 & 40 & 20 & 206 & 83 \\
\hline Kankan & 5 & 39 & 43 & 16 & 222 & 75 \\
\hline N'Zérékoré & 6 & 64 & 112 & 80 & 340 & 132 \\
\hline TOTAL & 34 & 251 & 307 & 226 & 1424 & 583 \\
\hline
\end{tabular}

La majorité des PPS visités (83\%) sont des centres de santé. D'autres types de PPS ont été également visités: les CHU de Donka et de Ignace Deen à Conakry, les 7 hôpitaux régionaux, 24 hôpitaux préfectoraux et 2 hôpitaux d'entreprise, les 5 cliniques de l'AGBEF et la clinique de Banankoro. La plupart de ces structures de santé $(97 \%)$ relèvent du secteur public et sont en milieu rural (59\%). 
Tableau $\mathbf{N}^{0} 2$ : Nombre d'observations SMI

\begin{tabular}{|l|c|c|c|c|}
\hline $\begin{array}{l}\text { Région } \\
\text { Naturelle }\end{array}$ & $\begin{array}{l}\text { Consultation } \\
\text { Prénatale }\end{array}$ & $\begin{array}{l}\text { Consultation } \\
\text { Post natale }\end{array}$ & $\begin{array}{l}\text { Consultation } \\
\text { MST/Sida }\end{array}$ & $\begin{array}{l}\text { Consultation } \\
\text { Enfant malade }\end{array}$ \\
\hline Conakry & 72 & 1 & 20 & 117 \\
Basse Guinée & 114 & 4 & 28 & 185 \\
Moyenne Guinée & 114 & 8 & 34 & 146 \\
Haute Guinée & 155 & 20 & 54 & 211 \\
Guinée Forestière & 251 & 34 & 82 & 295 \\
\hline TOTAL & $\mathbf{7 0 6}$ & $\mathbf{6 7}$ & $\mathbf{2 1 8}$ & $\mathbf{9 5 4}$ \\
\hline
\end{tabular}

2- Le volet sur les enfants malades.

\section{Graphique $\mathbf{N}^{\circ} 1$}

Répartition des enfants malades observés selon le type de PPS

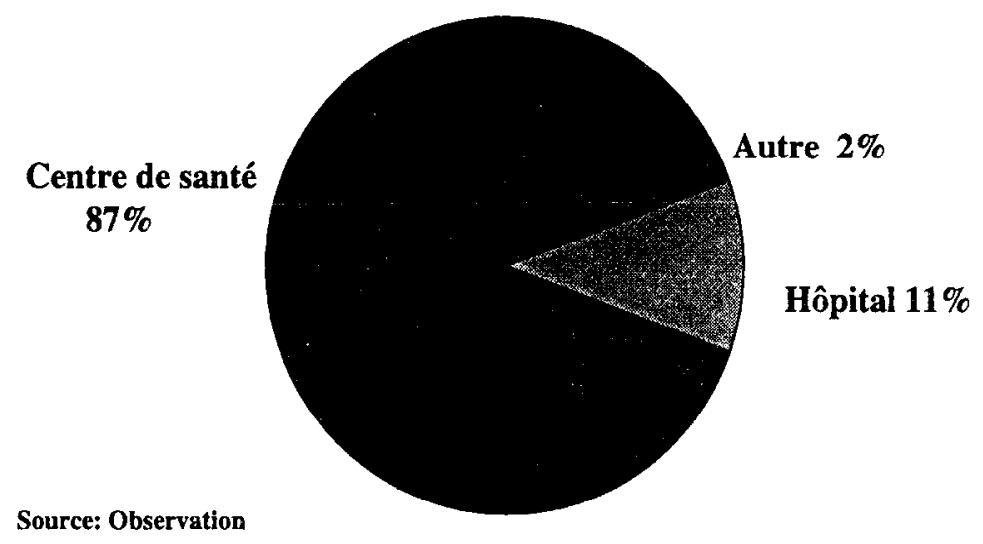

Dans le cadre de la survie de l'enfant, 954 enfants malades

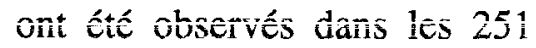
points de prestations de services visités. Seuls les enfants malades de moins de 5 ans ont été observés. Les enfants malades de 0 à 11 mois représentent $37 \%$ de l'échantillon. La pyramide d'âges des enfants malades de toux/rhume, difficultés respiratoires, diarrhée, fièvre, paludisme, anémie ou malnutrition montre une légère prédominance des enfants de 5 à 10 mois.

La majorité des enfants (87\%) ont été vus dans les centres de santé. In faut préciser que $54 \%$ de ces enfants vivent en milieu rural (54\%).

A la sortie de la consultation de l'enfant malade, la mère ou la personne accompagnante a été soumise à une interview. Sur les 953 mères ou accompagnantes, 705 ont pu être interviewées à la sortie soit une déperdition de $26 \%$.

Par ailleurs la répartition régionale des enfants malades observés montre une prédominance de N'Zérékoré $(26 \%)$. Les régions administratives les plus faiblement représentées sont Mamou $(6 \%)$, Boké $(7,5 \%)$ et Labé $(7,6 \%)$. Ce constat s'explique en partie par le nombre relativement plus faible de structures visitées dans ces régions. 


\section{Profil des clientes PF et SMI}

\subsection{Caractéristiques des clientes PF et SMI.}

Les clientes SMI sont relativement plus jeunes que les clientes de PF. L'âge moyen des clientes de SMI est de 25 ans et celui des clientes de PF est de 30 ans. L'étude montre également que $96 \%$ des clientes de SMI sont mariées contre $77 \%$ des clientes de PF. Il s'agit en général de mariage monogamique avec cependant une forte proportion de polygames (plus de $35 \%$ des clientes). La proportion de femmes scolarisées est de $39 \%$ pour les clientes de $\mathrm{PF}$ et de $22 \%$ pour les clientes de SMI. La majorité des clientes de PF scolarisées ont le niveau secondaire. Elles pratiquent pour la plupart la réligion musulmane ( $76 \%)$.

\subsection{Indicateurs de fécondité chez les clientes SMI et PF}

\section{Tableau No 3: Indicateurs de fécondité chez les cilentes $\overline{\mathrm{SM}} \overline{\mathrm{I}}$ et $\overline{\mathrm{P}} \overline{\mathrm{F}}$}

\begin{tabular}{|c|c|c|}
\hline Caractéristiques & Clientes PF & Clientes SMI \\
\hline Nombre d'enfants & $\begin{array}{c}\text { Pourcentage } \\
(\mathrm{n}=\mathbf{2 2 2})\end{array}$ & $\begin{array}{c}\text { Pourcentage } \\
(n=1410)\end{array}$ \\
\hline 0 & 11 & 15 \\
\hline $1 \cdot 3$ & 33 & 60 \\
\hline $4-6$ & 45 & 21 \\
\hline $7 \mathrm{et}+$ & 11 & 4 \\
\hline Nombre moyen/médian & ( 4 enfants) & ( 2 enfants) \\
\hline Minimum/Maximum & $0 / 12$ & $0 / 12$ \\
\hline Allaitement & & \\
\hline Oui & 14 & 36 \\
\hline Age dernier né & $(\mathrm{n}=199)$ & $(n=1378)$ \\
\hline$<6$ mois & 5.5 & 25.7 \\
\hline $7-18$ mois & 9 & 24 \\
\hline $19-24$ mois & 19.6 & 10.7 \\
\hline $25-36$ mois & 33.2 & 21.5 \\
\hline 37 mois et $t$ & 32.7 & 18.1 \\
\hline Age moyen/médian & (35 mois $/ 31$ mois) & (27 mois $/ 21$ mois) \\
\hline $\begin{array}{l}\text { Désir d'enfants } \\
\text { supplémentaires }\end{array}$ & $(n=222)$ & $(n=1422)$ \\
\hline Oui & 40 & 73.5 \\
\hline Non & 56 & 16.2 \\
\hline Dieu/Mari/Indécise & 4 & 10.3 \\
\hline Délai souhaité & $(\mathrm{n}=88)$ & $(n=1152)$ \\
\hline$<1$ an & 4.5 & 33.2 \\
\hline $1-2$ ans & 22.7 & 7.6 \\
\hline Plus de 2 ans & 36.4 & 27.8 \\
\hline Autre & 29.5 & 20 \\
\hline NSP & 6.8 & 11.5 \\
\hline
\end{tabular}

Les indicateurs montrent que la fécondité est plus élevée chez les clientes de PF. En moyenne les clientes de PF ont 4 enfants contre une moyenne de 2 enfants chez les clientes de SMI. Ce nombre moyen d'enfants par femme est inférieur à la moyenne nationale qui est de 5,7 enfants par femme (EDS, 1992). L'on constate cependant qu'il y a près de $12 \%$ de clientes de PF qui ont plus de 6 enfants (le maximum étant de 12 enfants). Parmi les clientes de PF, 14\% allaitent. Chez les clientes de SMI, environ une sur trois allaite et l'âge médian du dernier enfant-né est de 21 mois.

L'analyse montre aussi une certaine volonté de limiter les naissances chez les clientes de PF. En effet, une cliente de PF sur deux ne désire plus avoir d'enfants. Par contre seulement $16 \%$ des clientes de SMI ne souhaitent plus d'enfants. Ce constat pourrait s'expliquer par la fécondité différentielle et la relative jeunesse des clientes de SMI. Parmi les clientes qui désirent avoir des enfants, 36\% des clientes de PF et $28 \%$ des clientes de SMI souhaitent attendre plus de deux ans avant d'avoir le prochain enfant. L'on constate cependant qu'une cliente de SMI sur trois souhaite avoir son prochain enfant avant un an. 


\subsection{Opinion et utilisation de la contraception par les clientes de PF et de SMI}

Deux clientes de SMI sur trois approuvent les couples qui utilisent une méthode de planification familiale pour éviter ou espacer une grossesse. Peu de clientes de SMI sont au courant de la disponibilité de méthodes de PF au PPS (18\%). Parmi les clientes de SMI, seulement 3\% utilisent actuellement une méthode de contraception avec leur conjoint. Les méthodes contraceptives actuellement utilisées sont la Pilule (72\%), l'injectable (16,3\%), la PF naturelle (7\%) et la MAMA $(4,7 \%)$.

La majorité des clientes de $\mathrm{PF}(63 \%)$ ont déjà discuté de $\mathrm{PF}$ avec leur partenaire. Les principales méthodes de PF utilisées sont la pilule et l'injectable et cela quel que soit le statut de la cliente (nouvelle ou ancienne). Ce sont les méthodes pratiquées par plus de $80 \%$ des clientes de PF.

\section{Profil des Prestataires de Services}

Tableau N $\mathrm{N}^{0} 4$ Caractéristiques socio démographiques des prestataires de services

\begin{tabular}{|c|c|c|}
\hline Age & Femmes $n=339$ & Hommes $n=244$ \\
\hline Moins de 30 ans & 5.3 & 3.7 \\
\hline $30-39$ & 36.6 & 35.6 \\
\hline $40-49$ & 54.9 & 54.6 \\
\hline 50 ans et + & 3.2 & 6.1 \\
\hline Age moyen/médian & 40.2 ans $/ 41$ ans & $40.9 \mathrm{ans} / 41$ ans \\
\hline Minimum/Maximum & $23 / 52$ & $20 / 59$ \\
\hline Situation matrimoniale & $(\mathrm{n}=339)$ & $(\mathrm{n}=244)$ \\
\hline Marié (e) monogame & 48,1 & 58.2 \\
\hline Marié (e) Polygame & 37,5 & 34,0 \\
\hline Celibataire/Jamais mariê & 3,0 & 6,6 \\
\hline Divorcé /Veuf(ve)/séparé(e) & 11,5 & 1,2 \\
\hline Pratique Contraceptive & $(=339)$ & $(n=244)$ \\
\hline Oui & 45,1 & 65,2 \\
\hline $\begin{array}{l}\text { Méthode utilisée par le } \\
\text { prestataire/partenaire }\end{array}$ & $(n=153)$ & $(n=159)$ \\
\hline Pilule & 26,8 & 27,0 \\
\hline DIU & 5,9 & 1,3 \\
\hline Injectable & 26,1 & 18,2 \\
\hline Préservatif & 17.0 & 49,1 \\
\hline Spermicides & 6,5 & 6,3 \\
\hline C.C.V & 3,3 & 0,6 \\
\hline Méthode naturelle & 22,2 & 14,5 \\
\hline MAMA & 2,6 & 2,5 \\
\hline
\end{tabular}

Tous les prestataires de services de santé de la reproduction présents le jour de l'enquête ont été interviewés soit 583 agents. Ces prestataires de services viennent de tous les 251 PPS visités au cours de l'enquête.

Le tableau $\mathrm{N}^{\circ} 4$ résume les principales caractéristiques socio-démographiques des prestataires de services. En majorité, ce sont des femmes (58\%). Ce personnel est âgé de 41 ans en moyenne. Il s'agit généralement de personnes mariées (88\%). Parmi les personnes mariées, en moyenne $36 \%$ sont polygames soit plus d'un prestataire sur trois. Environ, $45 \%$ des prestataires femmes et $65 \%$ des hommes utilisent une méthode de contraception. Les méthodes de PF qu'ils pratiquent le plus sont le préservatif $(33 \%)$, la pilule (27\%), l'injectable (22\%) et la méthode naturelle $(18 \%)$. 


\section{B. ACCESSIBILITE AUX SERVICES DE SANTE DE LA REPRODUCTION}

L'accessibilité aux services comprend l'accessibilité en terme de services offerts, l'accessibilité géographique et l'accessibilité financière.

Tableau $\mathbf{N}^{\circ} 5$ : Disponibilité des services dans les PPS visités

\begin{tabular}{|l|c|}
\hline \multicolumn{1}{|c|}{ Services offerts } & $\begin{array}{c}\text { Pourcentage de PPS } \\
(\mathbf{n}=\mathbf{2 5 1})\end{array}$ \\
\hline 1- Planification familiale & 89,2 \\
2- IEC/PF & 87,6 \\
3- SR/PF hommes & 60,2 \\
4- SR/PF adolescents & 68,5 \\
5- Consultation stérilité/infécondité & 48,2 \\
6- Accouchements & 89,2 \\
7- Consultation prénatale & 92 \\
8- Consultation post natale & 92,4 \\
9- Soins post abortum & 69,7 \\
10- Vaccination & 84,9 \\
11- Surveillance nutritionnelle. Et pondérale & 77,7 \\
12- Récupération nutritionnelle & 43,0 \\
13- IEC/nutrition & 80,9 \\
14- Conseils allaitement maternel & 94,8 \\
15- Lutte contre les maladies diarrheíques & 97,6 \\
16- Infection Respiratoire Aiggue & 97,2 \\
17- Paludisme enfant & 97,6 \\
18- IEC VIH/Sida & 81,3 \\
19- Tests VIH/Sida & 11,6 \\
20- IEC MST autres que Sida & 94,0 \\
21- Diagnostic MST autre que Sida & 89,6 \\
22- Traitement MST & 92 \\
23- Services MST/Sida Adoiescents & 55 \\
24- Services MST/Sida Hommes & 52,6 \\
\hline
\end{tabular}

\section{Services offerts dans les PPS}

Les résultats de l'étude montrent que le paquet minimum de services n'est pas encore disponible dans tous les PPS. Cependant la majorité des PPS disposent de la plupart des services. En dehors des services de SR pour hommes et les adolescents, des tests de VIH/SIDA, les services de nutrition, de soins post abortum et de consultation pour stérilité. Tous les autres services sont habituellement disponibles dans plus de $80 \%$ des PPS visités.

Les données montrent que l'IEC en matière de $\mathrm{PF}$ est disponible dans $88 \%$ des PPS, niveau légèrement inférieur à l'IEC sur les MST.

\section{Accessibilité géographique}

En se référant aux interviews des clientes de SMI, il faut en moyenne 47 mn pour arriver au PPS. La duree médiane nécessaire pour se rendre à ce PPS est de $30 \mathrm{mn}$ et la durée maximale est de $60 \mathrm{mn}$. Ces résultats

montrent que malgré les énormes efforts consentis pour rendre les services de santé les plus proches possibles de la population, ceux-ci restent encore difficilement accessibles du moins géographiquement, surtout pour certains districts enclavés.

\section{Heure d'ouverture et durée de fonctionnement}

Selon la méthodologie de l'étude, les équipes de collecte étaient présentes dans les PPS avant l'heure d'ouverture officielle jusqu'au moment de la fermeture pour observer l'organisation quotidienne des services. Il a été constaté qu'environ 29\% des PPS ont débuté les prestations de service à l'heure. Les autres PPS ont accusé des retards dans le début des prestations des services, parfois de plus d'une heure. Plus d'une heure de retard a été observée dans 15\% des PPS. Un tel retard a été constaté dans des hôpitaux (21\%), dans des centres de santé (14\%) et dans une 
clinique AGBEF. Il est évident qu'un tel retard aurait pour conséquence de limiter l'accès des populations aux services surtout de PF.

\section{Coût des services}

Les coûts des services calculés à partir des données d'interviews varient considérablement selon le type de PPS. La consultation pour la PF coûte au maximum $1000 \mathrm{FG}$ à l'AGBEF et $5000 \mathrm{FG}$ dans le secteur public alors que le prix officiel est de 600FG. Quand aux produits de PF, leurs prix varient considérablement selon le type de PPS. Dans les centres de santé, c'est surtout en milieu urbain qu'il y a une surtarification des produits de PF. La pilule est parfois vendue à plus de 5000 FG dans certains centres de santé notamment à Conakry, Koundara, Mali, Beyla et Macenta.

En moyenne les coûts des services varient selon le type de clients. La consultation coûte en moyenne 1148 FG pour une cliente de PF, 933 FG pour une cliente de SMI et 433 FG pour un

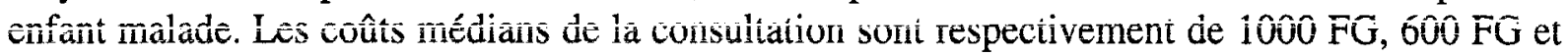
200 FG pour les clients de PF, de SMI et pour enfant malade.

L'étude a montré que la majorité des clientes de PF (80\%) et de SMI (74\%) ont trouvé les tarifs acceptables. Cependant, plus de 3\% des clientes de PF et $7 \%$ des clientes de SMI pensent que ces tarifs sont élevés.

La tarification des produits de PF pourrait être un facteur limitant l'accès des populations aux services. En principe, les produits contraceptifs, subventionnés par les bailleurs de fonds, devraient être vendus à des prix homologués. 
1. Des efforts doivent être consentis dans le sens de lextension du paquet minimume de services à tous les PPS. Un accent plus accru devrait être mis sur lamélioration de la disponibilité des services aux hommes et aux adolescents, les services de nutrition, les services pour stérilite et les soins post abortum.

2. Le respect des heures d'ouverture et de fermeture ainsi que la sensibilisation de la population sur les heures étendues de disponibilité des services contribueraient au désengorgement des structures de santé durant la matinée.

3. Une harmonisation des prix des consultations et des produits est nécessaire ainsi que laffichage des tarifs officiels dans les PPS. 


\section{C- CAPACITE FONCTIONNELLE}

Un des objectifs de l'étude c'est de contribuer à l'amélioration des services offerts par les PPS. Il s'agit dans cette section de déterminer la capacité fonctionnelle des PPS à fournir des services. Cette évaluation portera sur la disponibilité des infrastructures, matériels et équipements, l'expérience et la formation du personnel, la disponibilité du matériel et des activités d'IEC, la disponibilité des produits, la tenue des statistiques et rapports d'activités dans les PPS, la gestion et la supervision.

\section{Infrastructure, matériel et équipement}

a) Infrastructure

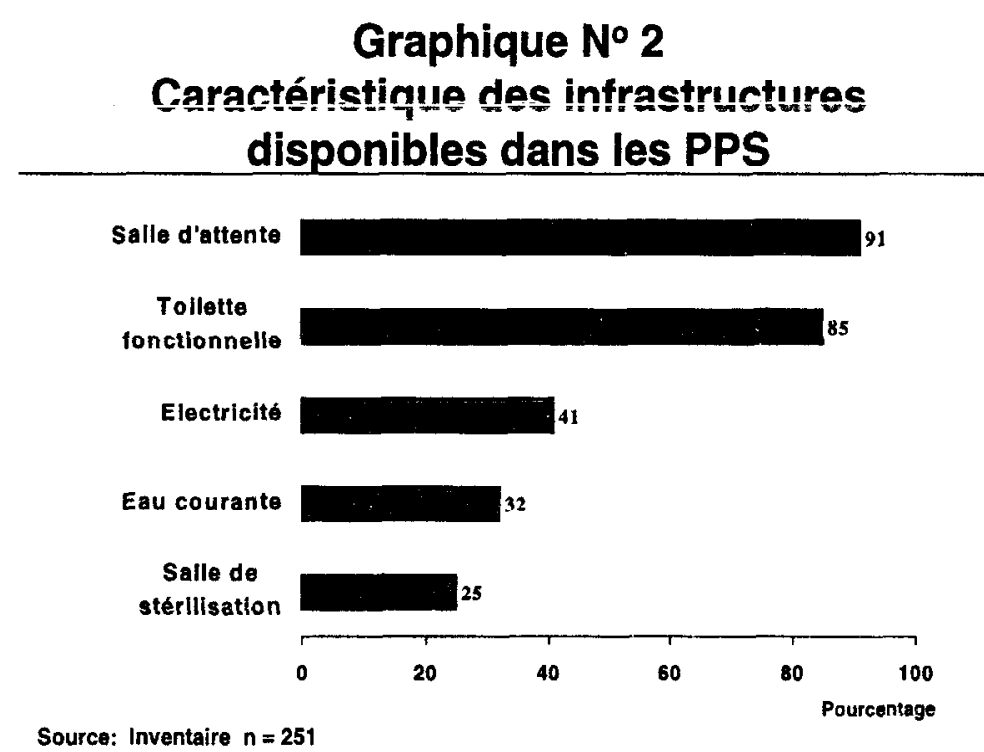

Dans le cadre de cette étude, les infrastructures ont été évaluées sur la base de l'existence d'une salle d'attente, d'une salle de consultation isolée, d'une salle de stérilisation, d'une toilette fonctionnelle et de la disponibilité de l'eau et de l'électricité.

Il existe une salle d'attente dans $91 \%$ des PPS. L'on trouve suffisamment de sièges dans seulement $61 \%$ des PPS. Des toilettes fonctionnelles ou des latrines existent dans $85 \%$ des PPS. Cependant ces toilettes ne sont que peu disponibles pour les client(e)s. La disponibilité de l'eau en quantité suffisante n'est pas une chose courante dans tous les PPS. En effet, sur 251 PPS visités, seuls 113 soit moins de la moitié, disposaient d'une quantité d'eau suffisante pour les activités du PPS. La situation est plus préoccupante en milieu rural où l'on ne trouve que 35\% de PPS pourvus de quantité d'eau suffisante contre $62 \%$ en milieu urbain. L'électricité est encore moins disponible en milieu rural (27\% des PPS ruraux et $62 \%$ des PPS urbains).

En ce qui concerne la consultation, celle-ci se déroule dans un endroit isolé dans $91 \%$ des PPS avec une discrétion auditive et visuelle dans plus de $90 \%$ des structures de santé visitées. Environ $81 \%$ des PPS disposent de locaux propres et 3 PPS sur 4 ont un éclairage adéquat.

\section{b) Equipement}

Un minimum d'équipement est nécessaire dans un PPS pour que les prestations puissent se dérouler dans de bonnes conditions. Les tables gynécologiques manquent dans $30 \%$ de PPS et $16 \%$ des PPS ne disposent pas d'un tensiomètre ou d'une armoire. Près de $27 \%$ des PPS manquent de gants tous types confondus. Les normes en SR prévoient que les structures de santé soient dotées d'un équipement minimum selon le niveau. Il apparaît que les centres de santé 
ayant 3 spéculums de différentes tailles ne sont pas nombreux. En effet, sur les 208 centres de santé visités, seulement $6 \%$ ont 3 spéculums grands modèles, $7 \%$ ont 3 spéculums petits modèles et $5 \%$ ont 3 spéculums de modèle moyen.

Quand on applique cette norme minimum à tous les PPS visités y compris les hôpitaux, il apparaît que seulement $6 \%$ des PPS ont au moins 3 spéculums de chaque modèle (grands,

Graphique $\mathbf{N}^{\circ} 3$

Proportion de PPS disposant de matériels techniques suivants

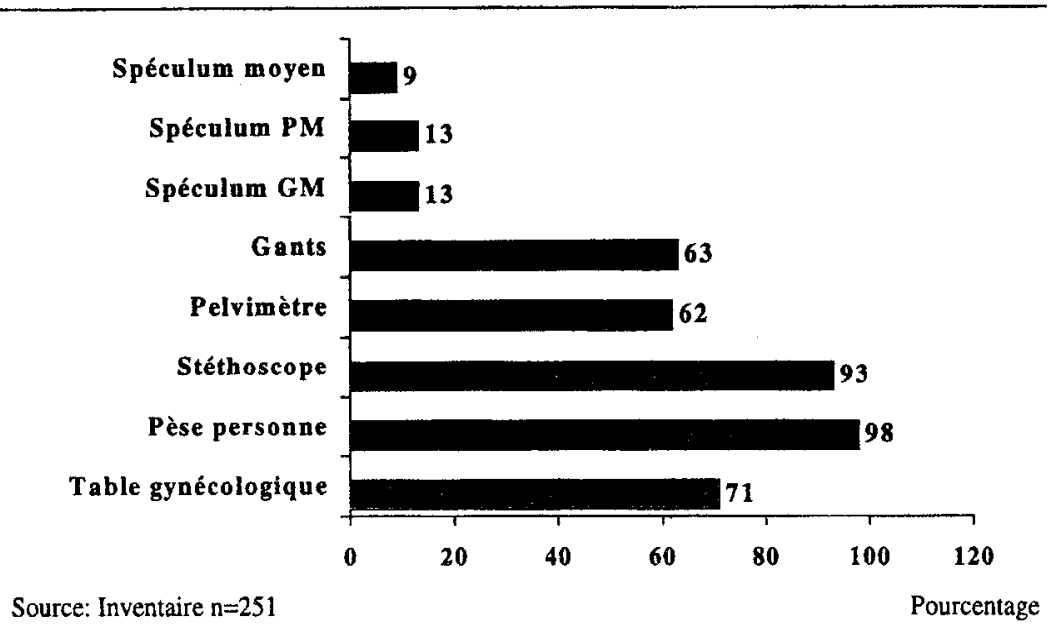
moyens et petits). Parmi les PPS qui offrent la méthode du DIU, environ $56 \%$ des PPS ruraux et $44 \%$ des PPS urbains n'ont pas un KIT complet de DIU. Les centres de santé sont les moins pourvus en table gynécologique (seulement $66 \%$ des PPS), en gants et en KIT de DIU. Il apparaît que les cliniques de l'AGBEF semblent disposer de plus de matériel pour la PF. Par contre, dans les régions, la situation varie en fonction de l'équipement. Les régions administratives de Kankan et

de N'Zérékoré sont celles qui manquent le plus de certains matériels importants pour des prestations de services dans le domaine de la santé de la reproduction. Par exemple, seuls respectivement $36 \%$ et $54 \%$ des PPS de ces deux régions ont une tabie gynécologique contre une moyenne nationale de $71 \%$. A Kankan, seulement $8 \%$ des PPS ont un KIT/DIU complet et $82 \%$ des PPS de cette région manquent totalement de gants.

Concernant le matériel de stérilisation, on a trouvé que $83 \%$ des PPS ont un stérilisateur à vapeur, $29 \%$ un autoclave, $20 \%$ un poupinel et $64 \%$ un système d'ébullition. Des différences importantes existent entre le milieu urbain et le milieu rural. En milieu rural, $94 \%$ des PPS ont le sterilisateur à vapeur et $12 \%$ le poupinel. Alors qu'en milieu urbain, le stérilisateur à vapeur existe dans $68 \%$ des PPS et le poupinel dans $30 \%$ des PPS.

Le matériel technique pour nourrissons manque dans presque tous les PPS de la Guinée. A Mamou, par exemple, près de 9 PPS sur 10 ne disposent pas de marteau à réflexes. Tout comme à Labé ; 93\% des PPS de Mamou n'ont pas d'extracteur de mucosités.

En général, le matériel de démonstration nutritionnelle n'est pas disponible dans les structures de santé. Dans la région de Labé, il n'y a pas un seul matériel de démonstration nutritionnelle. A Mamou et à Kankan, la situation est très critique dans ce domaine.

Les données de l'Analyse Situationnelle relatives à l'équipement indiquent que la majorité des PPS n'ont pas les normes requises. 


\section{Nombre, Expérience et formation du personnel dans les PPS}

\section{a) Nombre et type de personnel}

Les données tirées du questionnaire inventaire montrent que 69\% des PPS visités n'ont pas de médecin titulaire. L'on trouve moins de sages-femmes, de techniciens de santé et d'infirmiers que d'agents technique de santé (ATS). Environ 1 PPS sur deux n'a pas de sage-femme alors que seulement $2 \%$ des PPS manquent d'ATS.

\section{Graphique $\mathbf{N}^{\circ} 4$ \\ Nombre moyen de prestataires titulaires selon la localité}

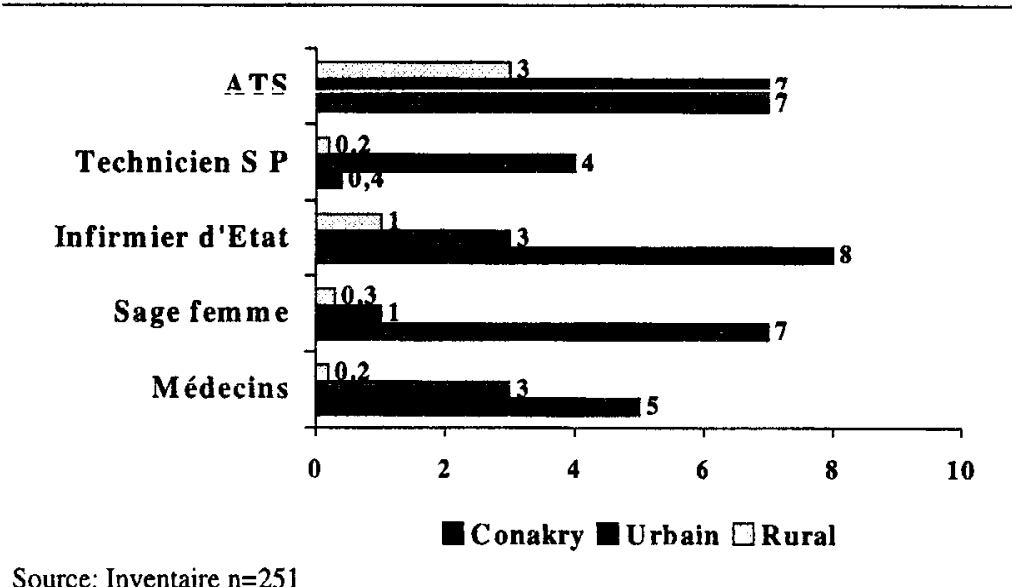

Si les effectifs des prestataires titulaires recensés au cours de cette étude étaient répartis en fonction du nombre de PPS, l'on aurait pu trouver en moyenne dans chaque PPS;

1 médecin, 1 sage-femme, 2 infirmiers et 5 ATS. Cependant, la répartition actuelle montre des disparités liées au milieu (urbain ou rural). L'on trouve en moyenne près de 5 Médecins par PPS à Conakry, contre 3 dans les autres villes et 0,2 en milieu rural. La situation est presque identique pour les sages-femmes: il y a près de 7

sages-femmes titulaires par PPS à Conakry contre 1 par PPS dans les villes de l'intérieur et 0,3 dans les sous-préfectures. Evidemment, ces résultats qui montrent des disparités entre les villes et le milicu rural méritent d'être examinées en fonction de la pyramide sanitaire.

Selon le type de PPS, on note que dans les hôpitaux nationaux, régionaux et d'entreprises, il y a en moyenne 7 médecins titulaires, 2 médecins stagiaires/bénévoles, 7 sage-femmes, 5 infirmiers et 8 ATS. Dans les hôpitaux préfectoraux, il y a en moyenne, 4 médecins titulaires, 1.7 sagefemmes, 3 infirmiers, 4 techniciens de santé publique et 6 ATS. Et dans les centres de santé et centres médicaux communaux, c'est en moyenne 0,9 médecins, 0.7 sage-femmes, 2.5 infirmiers, 1.7 technicien santé publique et 4.5 ATS. Il faut surtout remarquer que les stagiaires/bénévoles sont nombreux dans les structures de santé de la Guinée.

\section{b) Expérience et formation du personnel}

\section{- Expérience}

D'après les interviews réalisées auprès des prestataires de services de $\mathrm{SR}$, les états de service dans le même PPS remontent en moyenne à 6 ans. Il y a environ $17 \%$ des prestataires de SR qui ont moins d'une année de pratique dans leur PPS actuel. 
Le nombre d'années de services passées par le prestataire dans un PPS est un indicateur de la mobilité de celui-ci. Cet indicateur varie selon qu'il s'agit d'un PPS urbain ou rural : il est de 4 ans en milieu rural et de 8 ans en milieu urbain. Environ $14 \%$ du personnel de la SR en milieu urbain ont plus de 15 ans d'expérience dans leur PPS actuel contre seulement 3\% en milieu rural. $\mathrm{Au}$ niveau national, en moyenne $8 \%$ des prestataires n'ont pas été mutés depuis plus de 15 ans.

Selon la région administrative, il y a une nette différence entre Conakry et les autres villes. En moyenne, les prestataires de Conakry ont passé 10 ans dans leur PPS actuel contre 7 ans à Kankan; 6 ans à Mamou, Faranah et Boké; 5 ans à Kindia et Labé et 4 ans à N'Zérékoré.

Le pourcentage du personnel qui offre des services de PF est de $69 \%$. Presque tous (98\%) ont

\section{Graphique $\mathbf{N}^{\circ} 5$}

\section{Pourcentage de prestataires ayant reçu une} formation de base ou continue

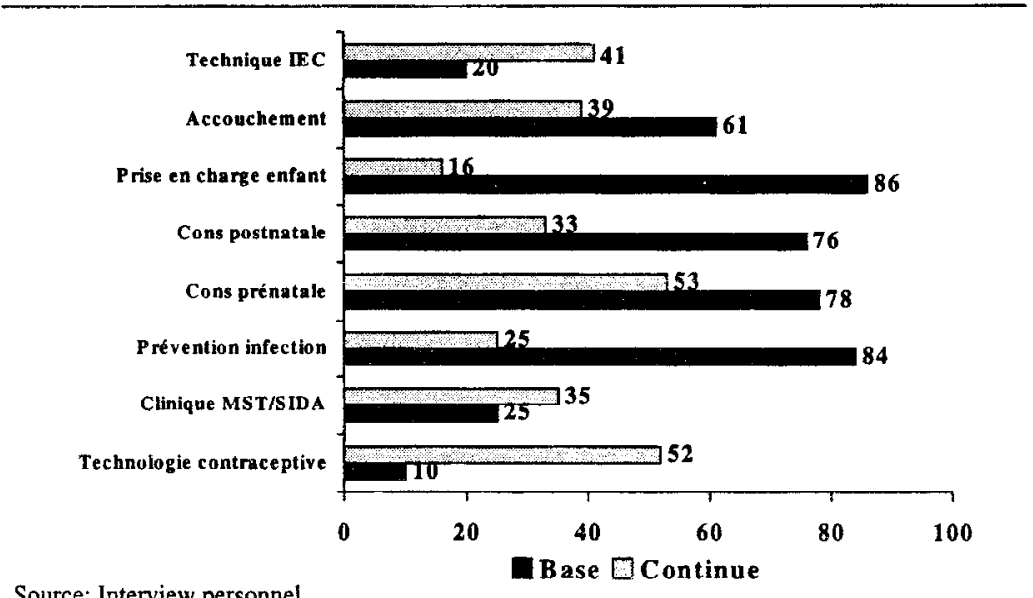

effectivement fournis des services de PF au cours des trois derniers mois précédant lenquête.

\section{- Formation}

Les résultats montrent qu'en moyenne, il s'est écoulé 12 ou 16 ans depuis que les prestataires actuels ont reçu leur formation de base. La formation de base en technologie contraceptive et sur les MST/SIDA date en moyenne de 12 ans alors que les autres

formations datent de 16 ans. Depuis lors, beaucoup de prestataires ont bénéficié de formation continue notamment en technologie contraceptive et en CPN (en moyenne 1 prestataire sur deux). Par contre, moins de $15 \%$ des prestataires interviewés ont été recyclés en consultation stérilité et en prise en charge des complications d'avortement. En moyenne ce recyclage remonte à 2,3 ou 4 ans selon le sujet.

Selon le type de personnel, on peut constater des différences dans les formations continues et dans les formations de base.

- les médecins : Sur les 91 interrogés, $85 \%$ ont reçu une formation de base en prénatal et $42 \%$ une formation continuc; $97 \%$ ont reçu une formation de base en prise en charge de l'enfant malade et $23 \%$ une formation continue; en Clinique MST/SIDA, ces pourcentages atteignent respectivement $29 \%$ et $51 \%$; et en technologie contraceptive, il y a $17 \%$ de formation de base et $45 \%$ de formation continue.

- Les sage-femmes : Sur les 111 interviewées, $98 \%$ ont une formation de base en prénatal et $52 \%$ une formation continue ; $78 \%$ une formation de base en prise en charge de l'enfant malade et $11 \%$ une formation continue; $11 \%$ une formation de base en technologie contraceptive et $58 \%$ une formation continue. 
- Les infirmiers ont surtout reçu une formation de base en prise en charge de l'enfant malade (94\%) et très peu en technologie contraceptive (5\%) et MST/SIDA (17\%); par contre 58\% ont reçu une formation continue en PF et $34 \%$ en clinique MST/SIDA.

- Les ATS sont surtout formés en prénatal (74\%) et en prise en charge de l'enfant (85\%) et très peu en $\mathrm{PF}(10 \%)$. La formation continue en $\mathrm{PF}$ a touché quand même $50 \%$ d'entre eux.

Le niveau de formation des prestataires présente une importance en ce sens qu'il témoigne de l'intérêt que le Ministère de la Santé accorde au développement de compétences techniques du personnel en SR. Il ressort de cette étude que des efforts accrus doivent être consentis dans le sens de la formation du personnel.

\section{Les activités et le matériel d'IEC}

\section{Graphique No 6 \\ Répartition des PPS selon le matériel IEC disponible par type de service}

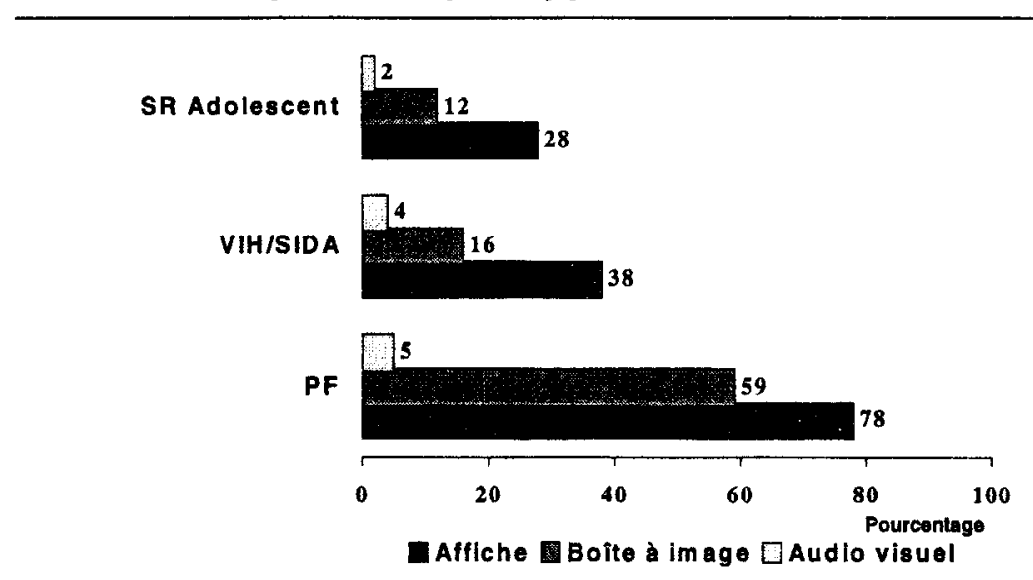

L'IEC est une composante essentielle de tout programme de SR. C'est un volet important pour l'utilisation des services de la santé de la reproduction par les populations guinéennes.

L'inventaire du matériel IEC indique qu'il n'est pas très disponible. En général, les affiches sont les plus utilisées et les autres matériels sont rares ou presque inexistants.

La PF et les MST/SIDA sont ceux qui bénéficient le plus de matériel IEC. Environ 4 PPS sur 5 ont des affiches sur la PF et plus de $30 \%$ des PPS ont des affiches sur les MST/SIDA. Par contre, on ne trouve des affiches sur la CPN ou le postnatal que dans $16 \%$ des PPS. On trouve également des dépliants et des boîtes à image dans quelques PPS surtout sur la PF ou les MST, mais presque pas de matériel audio-visuel (moins de 6\% des PPS).

La moitié des hôpitaux préfectoraux ont des boîtes à image en $\mathrm{PF}$, mais par contre aucune portant sur les soins prenatals ou la vaccination. Pour les centres de santé et CSM, c'est 60\% des PPS qui ont des boîtes à image sur la PF, $5 \%$ sur les soins prénatals et $2 \%$ sur la vaccination.

Une causerie éducative a eu lieu le jour de l'enquête dans $21 \%$ des PPS intégrés en SR/PF. C'est dans $8 \%$ des PPS qui offrent des services de PF qu'il y a eu une causerie sur la PF le jour de la visite.

Des panneaux ou des signes visibles annonçant que la PF est offerte dans le PPS existent dans $71 \%$ des PPS intégrés. Ces signes ou panneaux sont visibles seulement à l'intérieur du bâtiment dans $37 \%$ des PPS. Dans $12 \%$ des PPS, ce signe est visible seulement à l'extérieur du bâtiment. 
Les PPS qui ont des signes visibles à la fois à l'intérieur et à l'extérieur du bâtiment font $21 \%$ des structures de santé intégrées. Les PPS qui offrent des services de PF et qui n'ont pas de signes visibles indiquant la PF sont à la fois en milieu rural (32\% des PPS ruraux) et en milieu urbain (24\% des PPS urbains). Selon le type de PPS, il faut signaler qu'aucun des deux hôpitaux d'entreprise visités n'a un signe visible sur la PF tout comme $27 \%$ des centres de santé et une clinique AGBEF.

Une étude antérieure (Population Council,1994) met en avant une divergence d'opinion sur l'opportunité de mettre des panneaux indiquant un PPS qui offre de la PF. D'après cette étude, la raison avancée par les personnes qui y sont opposés, c'est que les femmes souhaitent garder l'anonymat, ce qui serait d'ailleurs l'une des principales raisons pour ne pas fréquenter le PPS le plus proche du domicile. Cependant en Guinée, sur $15 \%$ des clientes de PF qui ne fréquentent pas le PPS le plus proche de chez elles, seulement $3 \%$ ont déclaré que la principale raison est le désir de garder l'anonymat. Cette question sur l'opportunité de signaler l'existence d'un service de PF devrait faire l'objet d'une rechcrche opératioññelle.

\section{La disponibilité des produits et la logistique}

- Offre des produits et disponibilité le jour de la visite

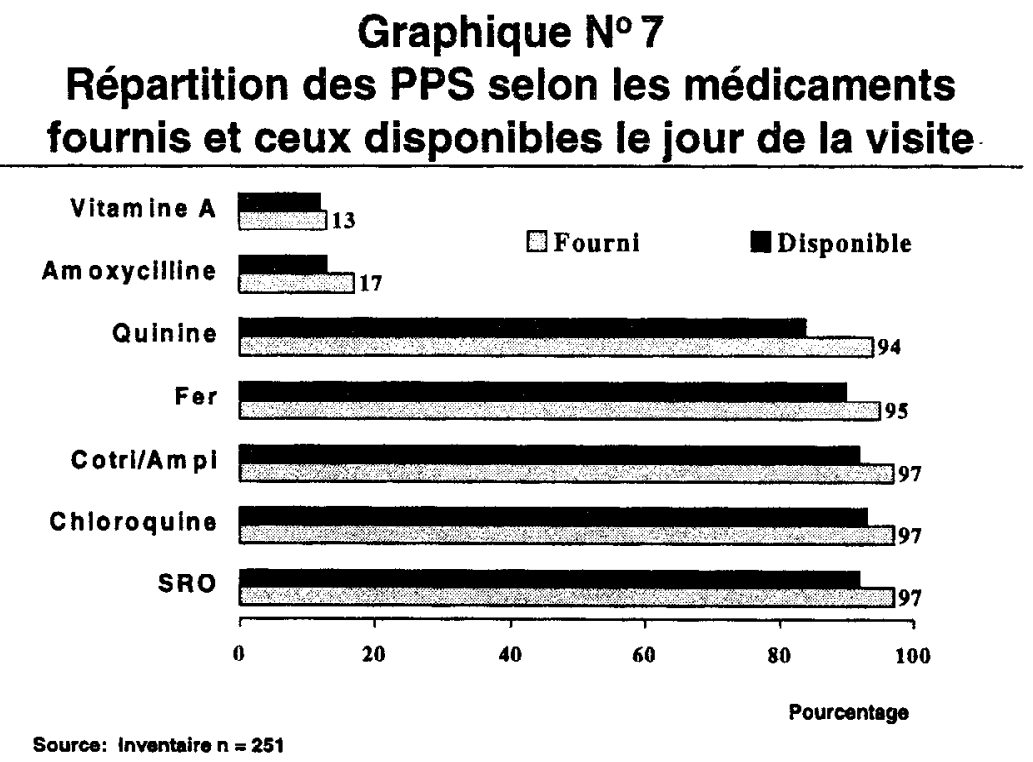

La pilule et l'injectable étaient offerts dans $83 \%$ des PPS. Les autres contraceptifs à savoir le spermicide, le DIU et le preservatif l'étaient dans respectivement $78 \%, 30 \%$ et $81 \%$ des cas. En général les produits contraceptifs étaient disponibles dans la majorité des PPS visités le jour de l'enquête. Cependant des ruptures de certains produits ont pu être constatés le jour de la visite. La pilule et les injectables étaient disponibles dans $97 \%$ et $90 \%$ des PPS qui ont déclaré offrir ces méthodes, le DIU et le

préservatif dans $90 \%$ et les spermicides dans $94 \%$ des PPS.

Les autres produits tels que la SRO, la chloroquine, le fer et la cotrimoxazole/ampicilline orale sont offerts dans presque tous les PPS. Cependant on peut noter la différence entre la proportion de produits habituellement fournis et celle des produits disponibles le jour de la visite (graphique 7). En effet c'est dans une proportion moindre de PPS que les produits ont étć trouvés le jour de la visite. 


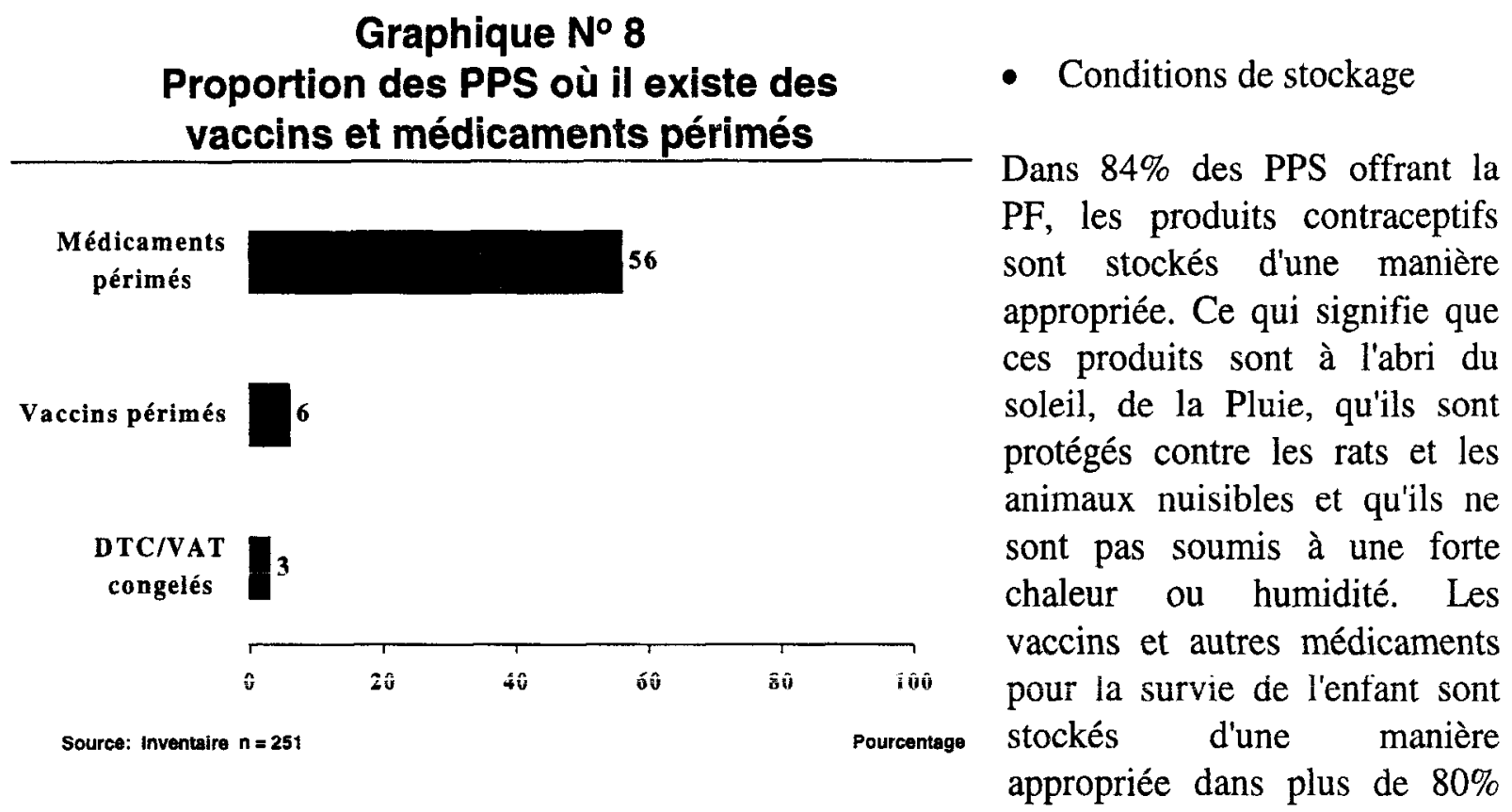

des PPS. Les médicaments pour les MST le sont dans seulement 77\% des PPS.

- Gestion des produits

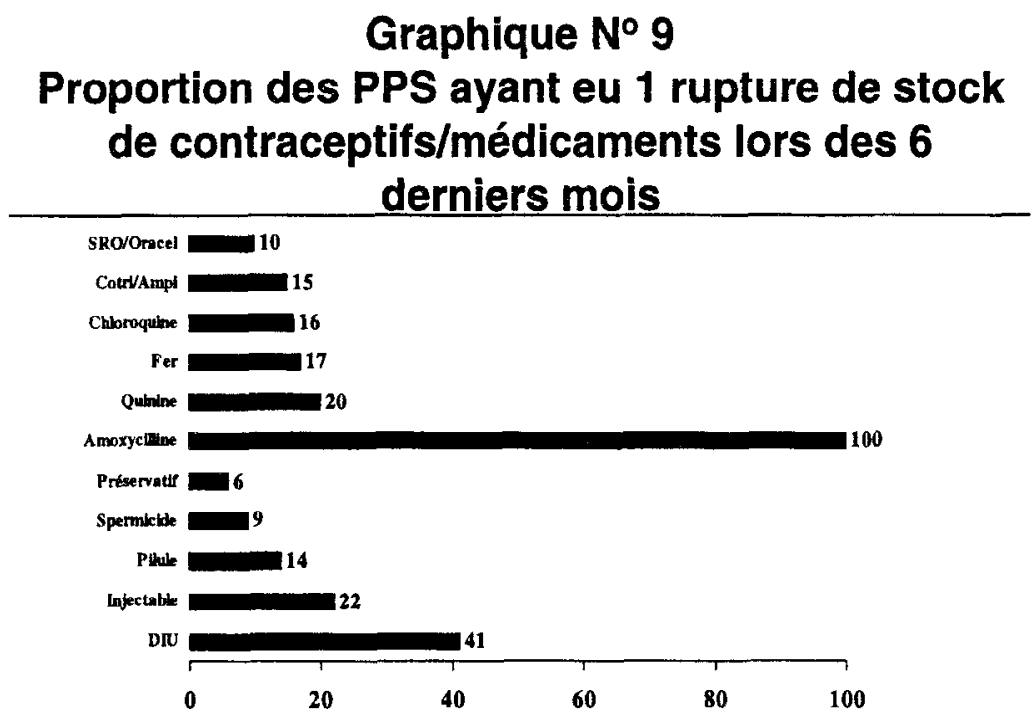

Source: Inventaire, $n=$ varie selon les produits

Pourcentage
Au cours de cette étude, il était aussi question de voir s'il existe un inventaire écrit des produits. Les données montrent que cet inventaire est surtout fait pour les maladies de l'enfant (96\% des PPS). Il existe un inventaire écrit des contraceptifs reçus dans $88 \%$ des PPS intégrés en PF. Pour les vaccins et les médicaments contre les MST, il y a un inventaire dans respectivement $84 \%$ et $81 \%$ des PPS visités. Le graphique No 9 montre qu'il y a effectivement des médicaments et des vaccins périmés dans les PPS. Dans

$56 \%$ des PPS visités, l'on a trouvé des médicaments périmés. Des vaccins périmés ont été trouvés dans $6 \%$ des PPS.

En outre, dans les 224 PPS du pays qui offrent des services de PF, 85\% ont déclaré avoir un système de commande de nouvelles provisions de contraceptifs; $60 \%$ de ces PPS font leur commande quand leur seuil d'alerte est atteint et $4 \%$ des PPS ne le font que lorsque le stock est épuisé. Ce seuil d'alerte varie d'un PPS à un autre. Les prestataires l'estiment parfois à travers le 
nombre de mois et souvent à 1 mois de la rupture (28\% des PPS). Il y a des PPS qui raisonnent en terme de quantité de médicaments encore disponibles (14\% des PPS) et enfin il y a des PPS qui ont un seuil d'alerte non défini (14\%). Les autres PPS font leurs commandes à plus d'un mois de la rupture ( 2 mois, 3 mois ou autres). Il y a un besoin d'harmonisation dans la définition de ce seuil.

L'étude s'est aussi intéressée aux ruptures de stocks au cours des 6 derniers mois. Il apparait qu'un nombre non négligeable de PPS ont connu des ruptures de stocks. Pratiquement tous les produits ont connu une rupture, y compris les vaccins. Ces ruptures vont du DIU avec $41 \%$ des PPS offrant la méthode, au SRO avec $10 \%$ des PPS. Il est à noter que la rupture en amoxycilline a touché tous les PPS. Ces résultats attestent de la nécessité de revoir le système de commande et d'approvisionnement des structures de santé.

\section{Les Statistiques et rapports d'activités}

\section{Graphique $N^{\circ} 10$ Proportion des PPS disposant des supports suivants pour le SNIS}

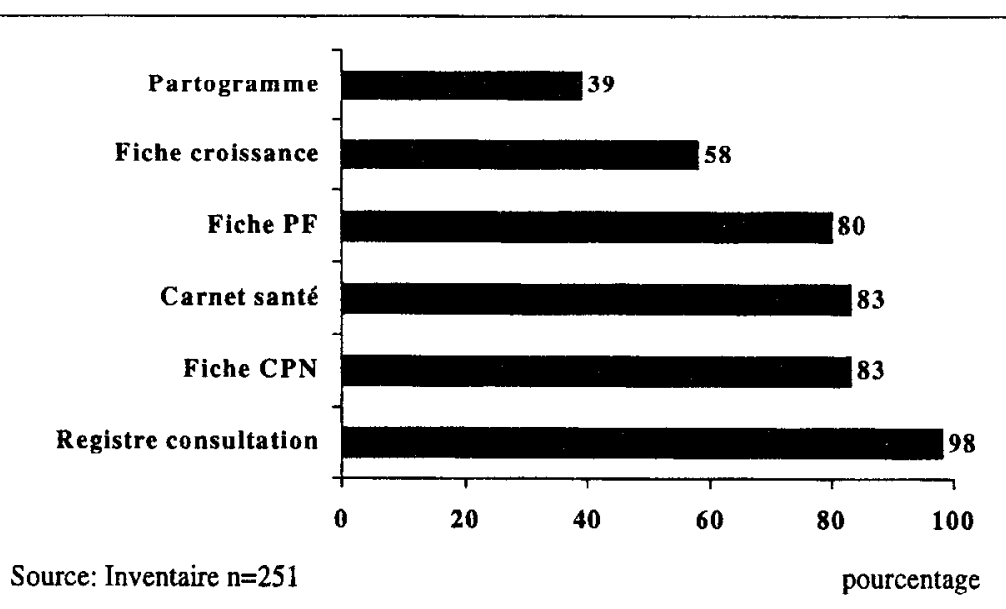

Pratiquement tous les PPS visités $\quad(99 \%) \quad$ enregistrent quotidiennement les activités de SMI/PF. Mais très peu de PPS (4\%) font un enregistrement journalier commun des activités de SMI/PF. Les PPS qui enregistrent tous les jours leurs activités de SMLPF sur des régistres séparés représentent $42 \%$. Les autres, la majorité des PPS $(53 \%)$ ne font des enregistrements que pour certaines activités. Les registres qui sont surtout en bon état sont ceux relatifs à la survie de l'enfant: IRA et paludisme (95\%), allaitement maternel

(94\%). Par contre, seulement $70 \%$ des PPS ont leur registre de PF bien tenu. Il y a donc beaucoup plus d'insuffisances dans la tenue des registres de PF que dans ceux de la SMI.

Les données montrent que les PPS envoient effectivement leurs rapports de statistiques mensuelles des activités à un superviseur ou à une unité supérieure (99\% des PPS). Environ $88 \%$ de ces PPS ont envoyé leur dernier rapport moins de deux mois avant l'enquête. Parmi ces PPS, seuls $37 \%$ ont eu un feed-back sur leur rapport. L'absence de feed-back sur la qualité des rapports et surtout sur l'utilisation qui en est faite pourrait décourager les prestataires dans la tenue des statistiques et dans l'élaboration de ces rapports. Les prestataires de services pensent que ces données pourraient servir fondamentalement à trois choses: évaluer, planifier, décider, améliorer la performance du PPS et la surveillance épidémiologique. 
Pour évaluer la performance des PPS, les registres et les cahiers ont servi d'outil au recueil de statistiques sur le nombre de clients servis d'une manière continue sur une période de 12 mois au cours des 24 derniers mois. Dans le cas où le PPS ne dispose pas de statistiques continues sur 12 mois, les statistiques de la période de disponibilité la plus longue ont été recueillies.

Les données tirées de l'inventaire des PPS montrent que la tenue des statistiques sur les activités de PF n'est pas systématique dans tous les PPS. Parmi les PPS offrant la PF, il y en a $24 \%$ qui n'ont pas de statistiques disponibles. Parmi ceux qui en ont, seulement $40 \%$ disposent de statistiques sur une période de 12 mois consécutifs. Pour les autres PPS, les données sont

\section{Graphique № 11}

Répartition des PPS selon le nombre de mois consécutifs où les statistiques de PF sont disponibles au cours des 24 derniers mois

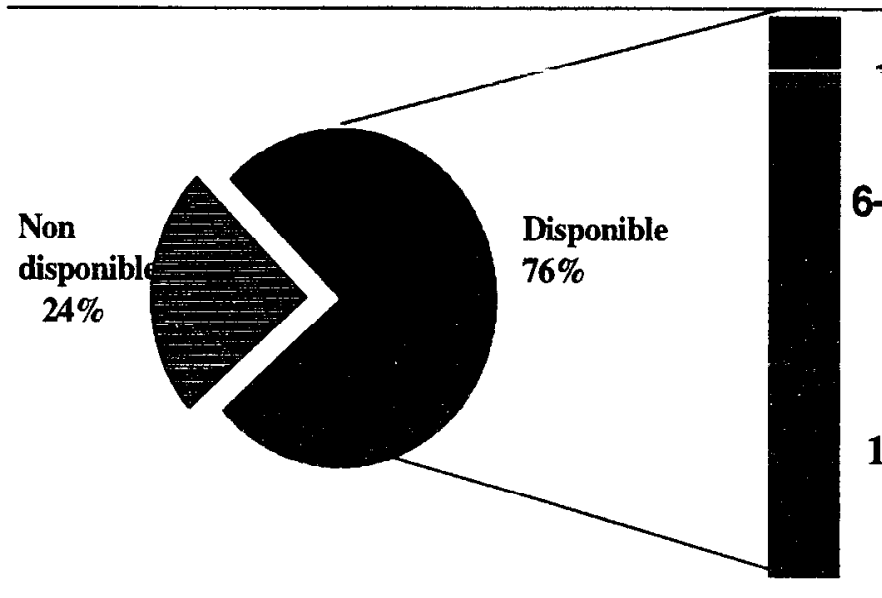

Source: Inventaire, $n=251$
Nombre de mois consécutifs où les statistiques PF sont disponibles $(n=186)$

disponibles sur des périodes variées:

- 19\% des PPS disposent de statistiques sur unc période de moins de 6 mois;

- 27\% des PPS ont des statistiques sur une période allant de 6 à 11 mois; et - 54\% des PPS en ont sur une période de 12 moins.

Parmi les facteurs qui déterminent la disponibilité des statistiques, figurent la localité, le type de

formation sanitaire et la région.

* Selon la localité, les résultats montrent qu'en milieu urbain $45 \%$ des PPS disposent de statistiques sur une période de 12 mois consécutifs contre $39 \%$ des PPS du milieu rural;

* Selon le type de PPS, les centres de santé comptent la proportion la plus importante de PPS disposant de statistiques sur 12 mois consécutifs (43\%) comparativement aux hôpitaux (26\%);

* Selon la région, la situation est relativement meilleure à Faranah (73\% de PPS disposent de statistiques sur 12 mois consécutifs) et Kankan (61\%). Par contre, il y a beaucoup moins de PPS avec des statistiques régulières à Conakry $(43 \%)$, N'Zérékoré $(36 \%)$, Kindia $(27 \%)$ et Labé $(26 \%)$. Dans les autres régions administratives, la situation est très préoccupante: Boké (17\%) et Mamou (13\%).

Les données recueillies dans les registres et autres documents statistiques des PPS, sous réserve de leur fiabilité, permettent de constater la faible utilisation des services de PF. En moyenne un PPS de la Guinée qui offre habituellement des services de PF n'enregistre que 86 clientes sur 12 
mois (le nombre médian est de 47 nouvelles clientes). Le nombre moyen d'anciennes clientes par PPS est de 240 (le nombre médian étant égal à 91). Selon la région, la moyenne varie considérablement. Conakry est la région qui enregistre le nombre moyen de clientes nouvelles comme anciennes le plus élevé. Pour ce qui concerne spécifiquement les nouvelles clientes, en dehors de Conakry, les régions administratives situées en Haute Guinée et en Guinée Forestière sont celles qui ont enregistré beaucoup plus de nouvelles clientes (au dessus de la moyenne). Par contre, s'agissant des anciennes clientes seules deux régions administratives ont plus de clientes que la moyenne nationale.

Selon le secteur, les statistiques recueillies à l'AGBEF montrent un nombre élevé de clientes nouvelles et anciennes (nettement au dessus de la moyenne nationale). Quel que soit le secteur considéré, l'on constate un écart assez important entre le nombre d'anciennes et de nouvelles clientes. Ce constat est également valable lorsqu'on observe les données selon le type de localité. Evidemment, le milieu urbain enregistre beaucoup plus de nouvelles clientes de PF (131 en moyenne par PPS par an) que le milieu rural (53 par an).

\section{Graphique № 12}

Répartition des PPS selon le nombre de mois consécutifs où les statistiques de CPN sont disponibles au cours des 24 derniers mois

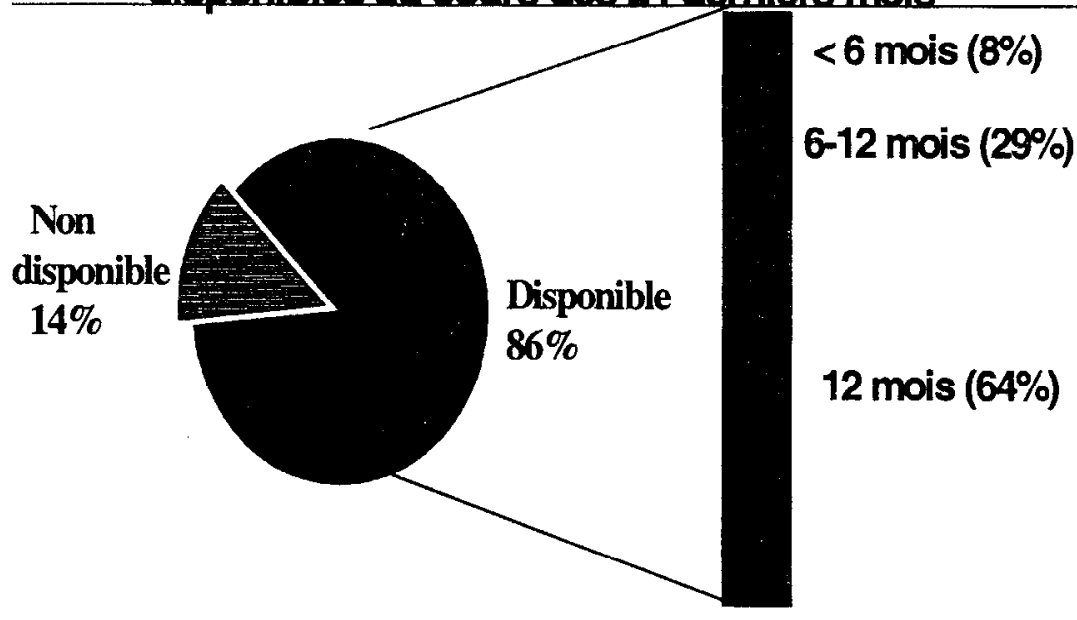

Source: Inventaire, $n=251$

Nombre de mois consécutifs où les statistiques CPN sont disponibles $(n=208)$
Concernant les consultations prénatales, les statistiques sont disponibles dans $86 \%$ des PPS. Seuls $14 \%$ n'avaient pas de statistiques. Parmi ceux ayant des statistiques, $8 \%$ en avaient sur une période de moins de 6 mois, 29\% sur une période variant entre 6 et 11 mois et $64 \%$ en avaient sur une période de 12 mois ou plus.

Comparativement à la $\mathrm{PF}$, un nombre relativement plus important de clients ont été enregistrés dans le cadre des soins prénatals et de la lutte contre les maladies diarrhéiques (LMD). En moyenne, l'on enregistre 669 nouvelles clientes et 724 nouveaux clients respectivement pour les soins prénatal et la LMD.

Les statistiques sur le nombre de clientes PF permettent d'évaluer les activités et l'état d'avancement du Programme de PF, de déterminer les besoins et d'établir des priorités d'action. Pour ce qui concerne le cas spécifique de la Guinée, il est important de préciser que ces statistiques de service auraient pu être plus fiables si toutes les clientes de PF étaient reçues dans les structures de santé. En effet, il a été signalé par le personnel de collecte que beaucoup de clientes de PF sont plutôt reçues à domicile par le prestataire responsable de la PF. Il semble que c'est pour des raisons de confidentialité d'une part mais aussi et surtout pour des raisons 
pécuniaires au bénéfice des prestataires. A cette cause de sous-évaluation des données sur la PF, il faut ajouter les besoins de formation et de motivation du personnel de santé sur le SNIS.

\section{La supervision}

Environ 3 PPS sur 10 collectent des informations pour le suivi et l'évaluation. La plupart des PPS bénéficient d'une supervision régulière (supervision centrale, régionale ou préfectorale). En effet, 92\% des PPS ont reçu au moins une supervision au cours des trois derniers mois. En moyenne ces PPS reçoivent 2 supervisions par mois. Au cours de ces supervisions certaines activités sont souvent accomplies.

\section{Graphique № 13 \\ Répartition des PPS (unités SR) seion le nombre de supervisions recues lors des 3 derniers mois}

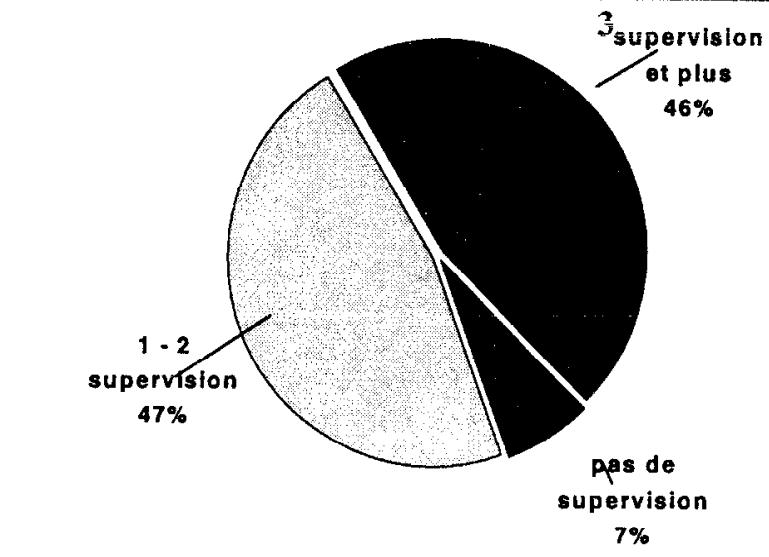

L'on constate que les principales activités menées lors de la supervision sont l'observation du service de SMI (76\%) et celle des soins dispensés dans les différents services (75\%). En moyenne seulement 1 PPS sur 2 a reçu une supervision relative à la PF au cours de la toute dernière supervision. On peut dire que si ce niveau de supervision est assez satisfaisant, le contenu de la supervision mérite d'être revu. Certains services reçoivent plus d'attention que d'autres.

\section{Conclusion}

L'infrastructure des PPS, même si elle atteint un niveau satisfaisant, montre encore quelques faiblesses dans certains éléments, en particulier la disponibilité en eau. Les toilettes ou latrines sont disponibles dans un nombre important de PPS, mais ne sont souvent réservées qu'au personnel. L'équipement minimum manque dans plusieurs PPS, particulièrement celui utilisé pour la prise en charge des enfants. Le personnel de SR a une moyenne d'âge élevée et n'est pas en nombre suffisant. L'offre des services s'appuit beaucoup sur les stagiaires et les bénévoles. La formation du personnel assez importante, cache cependant des inégalités selon les PPS et le niveau de la pyramide sanitaire.

L'IEC est un volet faible du programme de SR, même si celle en PF semble être plus disponible. D'importantes ruptures de stock ont été constatées, ce qui montre les lacunes du système de gestion des produits.

L'envoi des rapports à une unité supérieure est respecté par les prestataires, cependant l'enregistrement des client(e)s dans les supports du SNIS n'est pas systématique. Les PPS reçoivent des visites régulières de supervision. Du fait de la faiblesse des sous systèmes tels la 
gestion des produits ou les statistiques de service, il apparait que la supervision ne s'intéresse pas à tous les sous systèmes.

\section{Recommandations sur la Capacité Fonctionnelle}

1. Le manque de disponibilité de l'eau et de lélectricité, particulièrement en milieu rural doit être amélioré.

2. Un gros effort doit être fait pour léquipement total des struetures de santé, particulièrement des centres de santé. La liste standard d'équipenent pour tous les domaines de la SR tel que recommandé par les normes est loin d'être respectée.

3. Le recrutement de personnel qualifíe et jeune s'avère une nécessité. Des recyclages suivant les nouvelles normes et procédures amélioreraient également le plateau technique.

4. Le programme IEC dans le domaine de la santé de la reproduction doit être renforcé au niveau du PPS.

5. Les ruptures importantes qui ont été observées montrent que le système de commande et d'approvisionnement en produit renferme plusieurs lacunes. Une harmonisation du système de commande, une révision des seuils d'alerte et la formation en gestion logistique sont une priorité.

6. Si le pourcentage de PPS ayant bénéficié d'une supervision est important, 11 apparait que du fait des ruptures des produits, de la faiblesse de l'enregistrement des activités dans les régistres et du niveau moyen de la qualité des soins, le contenu de cette supervision doit être revu et amélioré. 


\section{D- QUALITE DES SOINS DES FEMMES}

L'Analyse Situationnelle de la Guinée examine la qualité des soins à partir de l'observation des consultations et des interviews avec les prestataires de service et les client(e)s. Les résultats sont présentés selon le modèle Bruce-Jain (1991) qui comprend six éléments: l'interaction prestataires-clientes, le choix de la méthode, les informations échangées entre le prestataire et les client(e)s, la compétence technique des prestataires, les mécanismes pour encourager la continuité et l'organisation appropriée des services.

\section{Relations Interpersonnelles}

Cet élément de la qualité des soins porte principalement sur le contenu des échanges et la convivialité entre le prestataire et la cliente depuis l'acceuil jusqu'à la sortie de la consultation. Il permet de mesurer la qualité des rapports humains ainsi que la perception des clientes des services reçus. Pour évaluer cette composante de la qualité, plusieurs indicateurs ont été utilisés.

Concernant l'accueil, il ressort de l'observation que les règles de bienséance ont été respectées dans la mesure où la quasi totalité des client(e)s ont reçu des salutations cordiales $(98 \%)$. Les données montrent que la plupart des client(e)s semblent satisfaits des services reçus et ont pu poser des questions $(94 \%)$. I faut cependant noter que la satisfaction du client saisie au sein de la structure entraîne un biais de coutoisie positif dans les réponses.

\section{Graphique No 14 Temps d'attente des clientes}

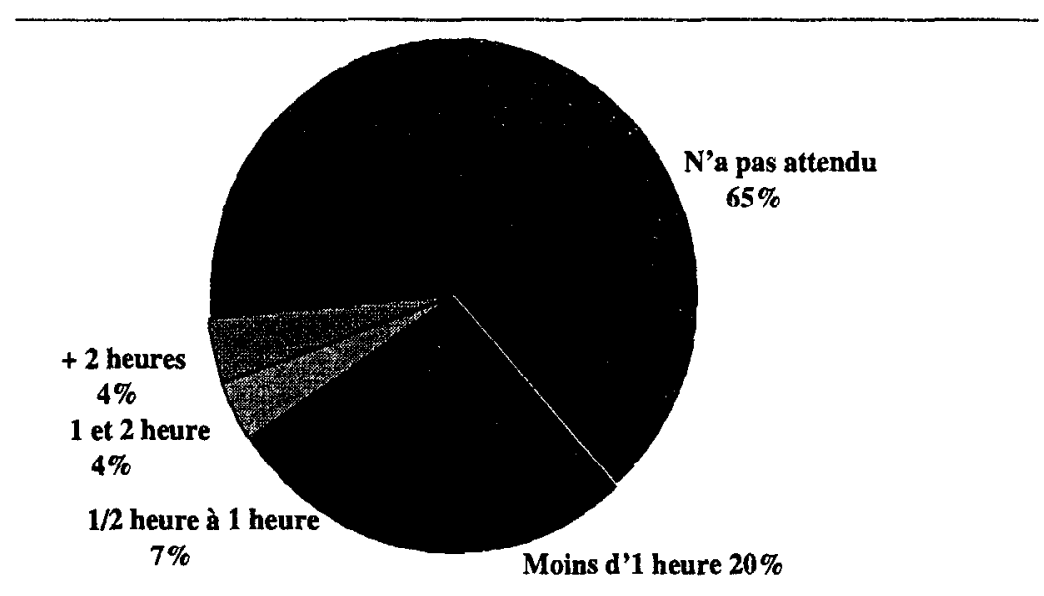

Pour une bonne appréciation de la qualité des soins, il est important de prendre en compte le temps d'attente au point de prestation de services. Dans la majorité des cas $(64 \%)$, les clientes de PF ont déclaré n'avoir pas du tout attendu avant d'être reçue. Les clientes de PF qui ont attendu plus d'une demi-heure parfois même plus de 2 heures de temps représentent $12 \%$.

Le temps consacré aux Source:interview cliente PF $\mathbf{n = 2 2 6}$ consultations de PF ou de SMI est très variable. La durée médiane des consultations des nouvelles clientes de PF est de $15 \mathrm{mn}$. Par contre le temps médian de consultation d'une ancienne cliente de PF est de seulement $10 \mathrm{mn}$. II faut préciser que la durée médiane de consultation la plus faible enregistrée au cours de cette étude est celle consacrée à un enfant malade soit $8 \mathrm{mn}$. Pour la moitié des patientes venues pour soins prénatals la consultation a duré 10 minutes. Pour les clientes de SMI, 45\% d'entre elles sont satisfaites de la durée de consultation. Par contre, $40 \%$ pensent que c'est trop court et $14 \%$ des clientes ont trouvé ce temps trop long. 


\section{Choix de la méthode de PF}

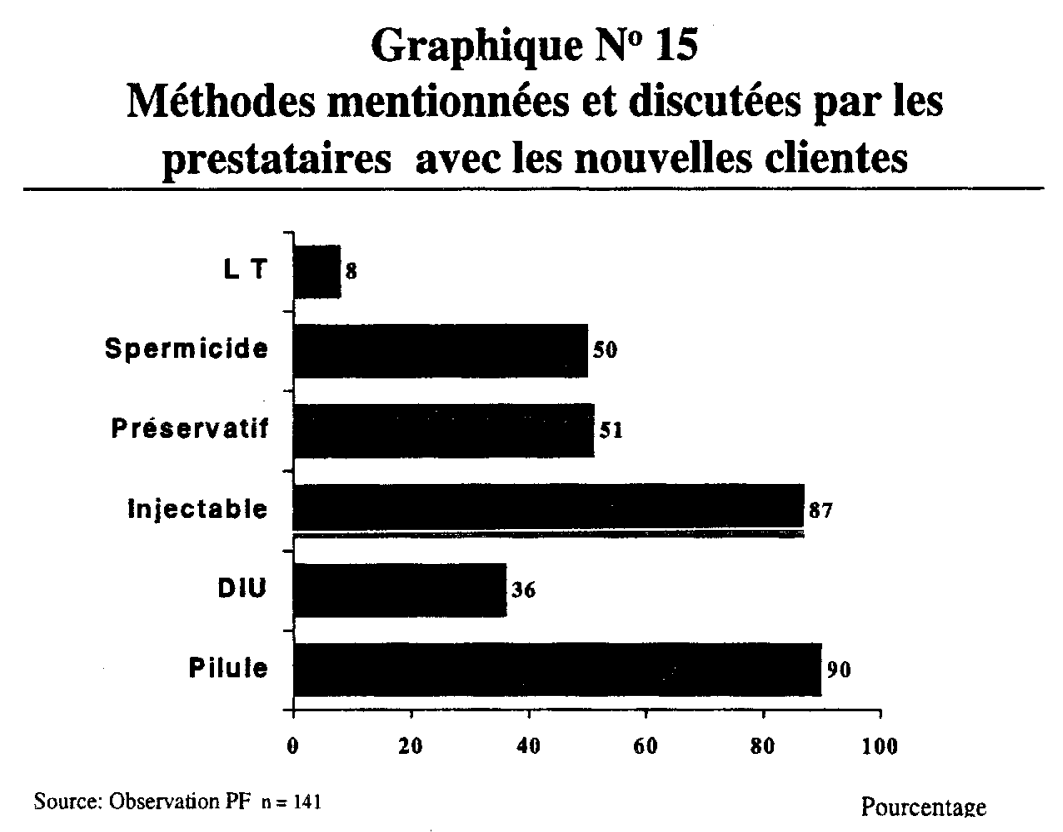

En Guinée, plusieurs méthodes contraceptives sont disponibles. L'on y trouve les méthodes barrières, les méthodes hormonales et les méthodes chirurgicales: Pilule, DIU, injectable, préservatif, spermicides, CCV. Bien entendu cette disponibilité dépend du niveau de la pyramide sanitaire.

Méthodes discutées avec les clientes. Les nouvelles clientes de PF ont été informées sur diverses méthodes de PF. Elles ont été informées surtout sur la $\mathrm{CCV}$, la pilule et l'injectable. Le préservatif, les spermicides et le DIU sont mentionnés dans une moindre mesure.

Environ $88 \%$ des nouvelles clientes de PF ont mentionné leur préférence pour une méthode contraceptive lors de la consultation, et $78 \%$ d'entre elles ont effectivement obtenu leur méthode. Cependant il apparaît que durant l'entrevue avec les nouvelles clientes, les prestataires influencent leur choix. En effet, les principales méthodes proposées aux nouvelles clientes par les prestataires sont la pilule et l'injectable. Ces deux méthodes sont recommandées par respectivement $90 \%$ et $87 \%$ des prestataires.

Les données montrent que $44 \%$ des clientes ont choisi d'utiliser la pilule et $43 \%$ les injectablcs. Les autres nouvelles clientes ont choisi la CCV ( 2 clientes), les spermicides ou/et préservatifs ( 9 clientes), le DIU (4 clientes). Les nouvelles clientes qui n'ont pas obtenu de méthode le jour de la visite sont au nombre de 6. Les interviews des nouvelles clientes de PF ont montré que les principales raisons pour lesquelles la méthode obtenue est parfois différente de celle préférée sont l'attente des menstruations (22\%), la rupture de la méthode (23\%) et les contre indications médicales (14\%). Par contre, chez les anciennes clientes, les raisons avancées sont essentiellement les effets secondaires (31\%), l'attente des menstruations (23\%) et la suspicion d'une grossesse (15\%).

Barrières à la contraception. Selon les normes en vigueur, la méthode contraceptive choisie par la cliente devrait lui être fournie en dehors de toute contre-indication médicale. Cependant on remarque que pour avoir accès à la contraception, les prestataires ont parfois des critères d'éligibilité basés sur l'âge, la parité, le statut matrimonial et le consentement du conjoint. 
Quelle que soit la méthode contraceptive considérée, l'on trouve des prestataires qui ont des restrictions concernant l'âge. Mais celles-ci portent beaucoup plus sur l'âge minimum que sur l'âge maximum d'éligibilité pour la contraception. En effet, c'est plus de $70 \%$ des prestataires interviewés qui en imposent un. Cette restriction touche même les préservatifs et les spermicides pour lesquels plus d'un prestataire sur trois exige un âge minimum. Cet âge minimum varie selon les méthodes. Il est de 15 ans pour la pilule et les injectables.

Pour offrir une méthode contraceptive, le personnel de santé s'intéresse aussi à la parité de leur cliente. La parité exigée pour accéder à une méthode contraceptive augmente avec le degré de reversibilité. Pour la pilule et le DIU, les prestataires exigent un minimum de 3 enfants. Avec les injectables ou la contraception chirurgicale, la parité minimale exigée est respectivement de 4 à 5 enfants.

Le statut de femme célibataire et le consentement du conjoint sont également des critères d'éligibiiite pour obtenir la contraception même si cela touche moins de prestataires. En effet, quelle que soit la méthode considérée il y a des prestataires qui n'offriraient pas la contraception à une célibataire et même à une femme mariée sans l'accord du conjoint.

Tableau $\mathbf{N}^{0}$ 6: Pourcentage de prestataires ayant des critères d'éligibilité

\begin{tabular}{|c|c|c|c|c|c|}
\hline $\begin{array}{c}\text { Restrictions des } \\
\text { Prestataires }\end{array}$ & $\begin{array}{c}\text { Age minimum } \\
\boldsymbol{\%}\end{array}$ & $\begin{array}{c}\text { Age maximum } \\
\boldsymbol{\%}\end{array}$ & $\begin{array}{c}\text { Minimum } \\
\text { enfant \% }\end{array}$ & $\begin{array}{c}\text { Statut femme } \\
\text { célibataire \% }\end{array}$ & $\begin{array}{c}\text { Consentement } \\
\text { du mari } \%\end{array}$ \\
\hline Pilule & 71.1 & 16.3 & 35.3 & 20 & 19 \\
\hline Préservatif & 35.0 & 25.6 & 15.9 & 19 & 42 \\
\hline Spermicides & 38.5 & 24.0 & 16.4 & - & - \\
\hline DIU & 65.5 & 62.0 & 48.2 & - & 29 \\
\hline Injectable & 78.4 & 62.5 & 53.6 & 26 & 21 \\
\hline CCV & 73 & 55.5 & 78.4 & 57 & 90 \\
\hline PF naturelle & 33.7 & 32.9 & 21.6 & - & - \\
\hline
\end{tabular}

Source: Interview Personnel, $n=371$

Méthodes recommandées selon les objectifs de la cliente. De facon générale, les prestataires distinguent les méthodes contraceptives d'espacement et de limitation des naissances. Pour retarder ou espacer les naissances en l'absence de contre-indications, ce sont la pilule, l'injectable, le DIU, le préservatif et le spermicide qui sont recommandés. Dans le cas où la cliente ne souhaiterait plus avoir d'enfants, c'est la contraception chirurgicale volontaire qui est recommandée. Par ailleurs, les prestataires ont déclaré qu'il y a des méthodes qu'ils ne recommanderaient jamais à leurs clientes. Cette catégorie de prestataires représente $11 \%$ du personnel de santé interviewé. La principale méthode que les prestataires ne recommanderaient jamais est la CCV. 
Tableau $\mathbf{N}^{0} 7$ : Méthodes recommandées selon les objectifs de la cliente

\begin{tabular}{|c|c|c|c|}
\hline Méthode contraceptive & $\begin{array}{c}\text { Pour retarder ou espacer } \\
\text { les naissances } \\
\mathbf{n}=\mathbf{( 3 6 8 )}\end{array}$ & $\begin{array}{c}\text { Pour limiter les } \\
\text { naissances (n=361) }\end{array}$ & $\begin{array}{c}\text { Ne recommande jamais } \\
\text { (n=39) }\end{array}$ \\
\hline Pilule & 92 & 7 & 15 \\
\hline DIU & 65 & 19 & 15 \\
\hline Injectable & 73 & 18 & 8 \\
\hline Préservatif & 62 & 4 & 3 \\
\hline Spermicide & 62 & 3 & 59 \\
\hline CCV & 3 & 94 & 5 \\
\hline PF naturelle & 42 & 3 & 3 \\
\hline Mama & 25 & 1 & 10 \\
\hline
\end{tabular}

Par contre, le DIU et la CCV sont les méthodes les moins proposées aux nouvelles clientes de $\mathrm{PF}$. On constate que $8 \%$ des prestataires recommanderaient la CCV à une nouvelle cliente de PF. Cette information laisse supposer que ces femmes doivent être des grandes multipares. Il faut aussi noter que $73 \%$ des prestataires de service pensent que les injectables peuvent causer l'infécondité ou la stérilité permanente. A la question de savoir s'ils recommandent systématiquement des examens de laboratoire avant l'offre de la contraception, 53\% des prestataires ont répondu affirmativement.

\section{Informations échangées entre les clientes de SR et les prestataires}

La qualité des soins mesurée par l'information échangée avec la cliente a été évaluée à partir de l'information relative aux questions posées par les prestataires au cours de la consultation avec les clientes, à l'information donnée par les prestataires aux clientes sur la nouvelle méthode acceptée, aux questions posées par les prestataires concernant la première visite prénatale ou pour MST/SIDA et enfin l'information retenue par les femmes.

\subsection{Concernant les visites prénatales.}

L'observation des consultations montre que conformément au protocole, l'interrogatoire est plus complet pour les premières visites que pour les visites subséquentes. L'observation des consultations

\section{Graphique № 16}

Questions abordées lors de la consultation prénatale

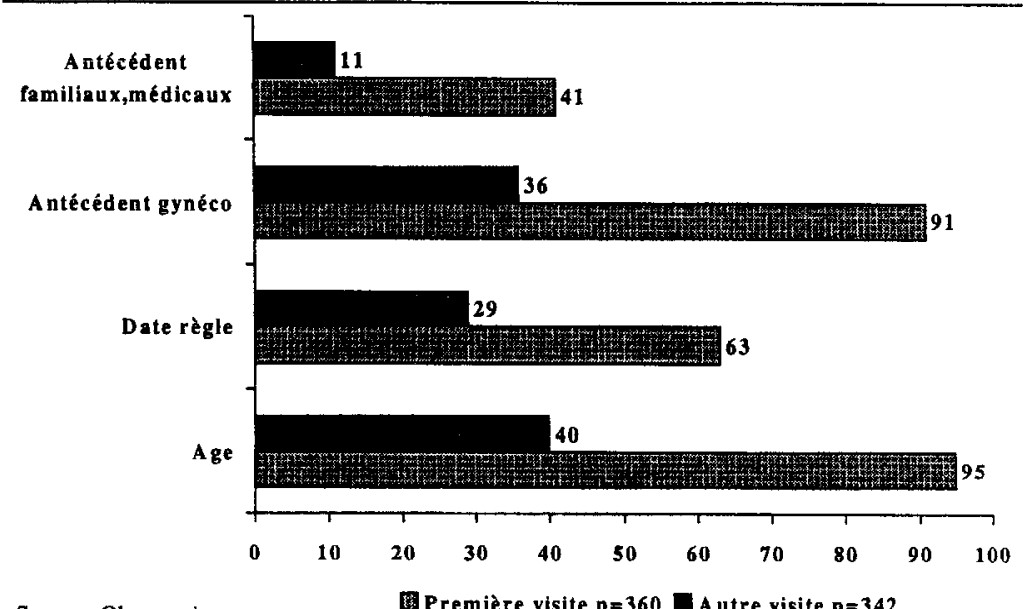

Source: Observation

Première visite $n=360$ A utre visite $n=342$ 
montrent que les prestataires se renseignent surtout sur l'âge de la patiente et les antécédents gynécologiques. Par contre la date des dernières règles et les antécédents familiaux et médicaux sont abordés dans une moindre mesure. Il en est de même pour le poids et l'âge du dernier né de la patiente

Peu d'explications et de conseils sont donnés aux clientes. Il apparaît que $44 \%$ des nouvelles clientes ont eu droit aux résultats de l'examen, $18 \%$ ont reçu des conseils sur l'hygiène et $3 \%$ des conseils sur l'allaitement maternel. Ces pourcentages chutent au cours des visites subséquentes.

\subsection{Concernant les consultations de MST/SIDA.}

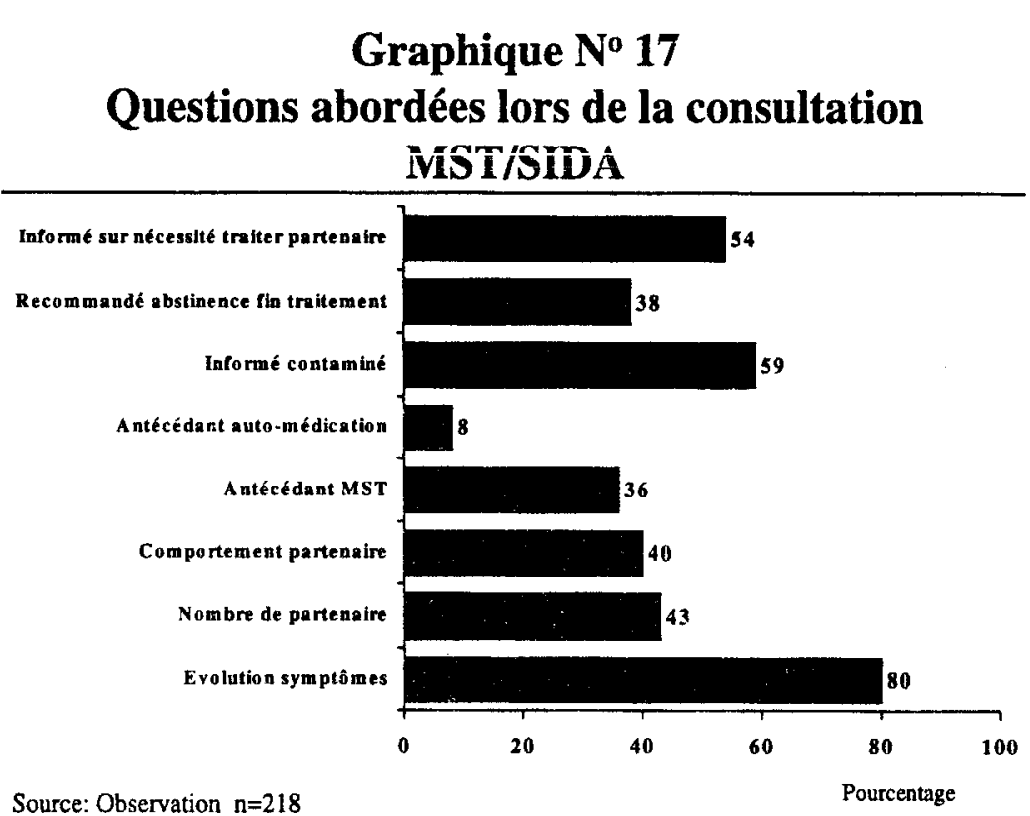

Les prestataires se préoccupent généralement de connaître l'évolution des symptômes. Les données montrent aussi que ce ne sont pas tous les prestataires qui informent la patient(e) du fait qu'il y a contamination et que le partenaire doit en être informé et être traité. Quand au comportement en matière de sexualité du patient ou de la patiente, les prestataires s'en informent dans une moindre mesure. En effet le nombre de partenaires, le comportement du partenaire, la recommandation de l'abstinence jusqu'à la fin du traitement et les antécédents de MST sont abordés avec moins de la moitié des clientes. Quand aux antécédents d'auto-médication, ils sont peu discutés. Seulement $12 \%$ des clientes ont eu à poser des questions.

\section{Graphique No 18}

Informations données aux clientes sur la nouvelle méthode acceptée

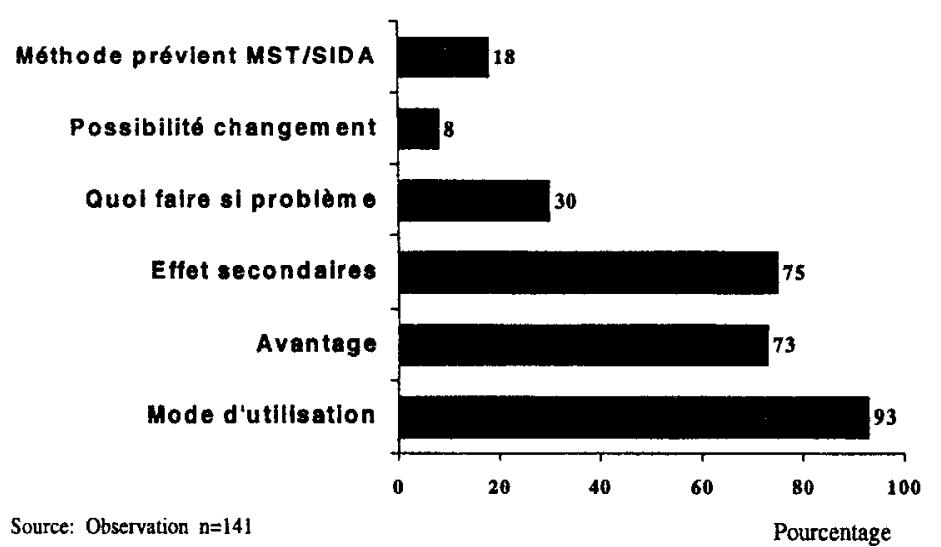

\subsection{Informations données aux clientes de PF.}

Les observations des consultations des clientes de PF permettent de voir les informations qui sont fournies à la cliente en cas d'acceptation d'une nouvelle méthode. En général, ce sont trois éléments parmi les 7 prévus dans les normes et procédures qui sont souvent abordés. En effet le mode d'utilisation, les inconvenients ou 
effets secondaires et les avantages sont présentés à la majorité des nouvelles clientes. Le counseling sur ce que devrait faire la cliente en cas de problème a été abordé dans le tiers des consultations. La possibilité pour la cliente de changer de méthode si celle qu'elle utilise ne lui convient pas a été abordée au cours de très peu de consultations, ainsi que la capacité ou non de la méthode à prévenir les MST/SIDA. Le matériel de démonstration le plus utilisé durant les consultations est l'échantillon de contraceptif. Avec $26 \%$ de nouvelles clientes aucun matériel pour faire le counseling n'a été utilisé. Les données font apparaitre aussi que les antécedents en matière de reproduction constituent le principal sujet presque toujours abordé au cours des consultations de PF (92\%). Les prestataires n'ont demandé qu'à $50 \%$ des nouvelles clientes leur intention en matière de reproduction. Environ une cliente de PF sur 5 n'a bénéficié d'aucune question concernant son cycle menstruel (régularité ou irrégularité). La question relative aux inquiétudes concernant les MST/SIDA n'est presque jamais abordée chez la nouvelle cliente de

\section{Graphique No 19}

\section{Connaissances de la contraception par les clientes utilisatrices de pilule}

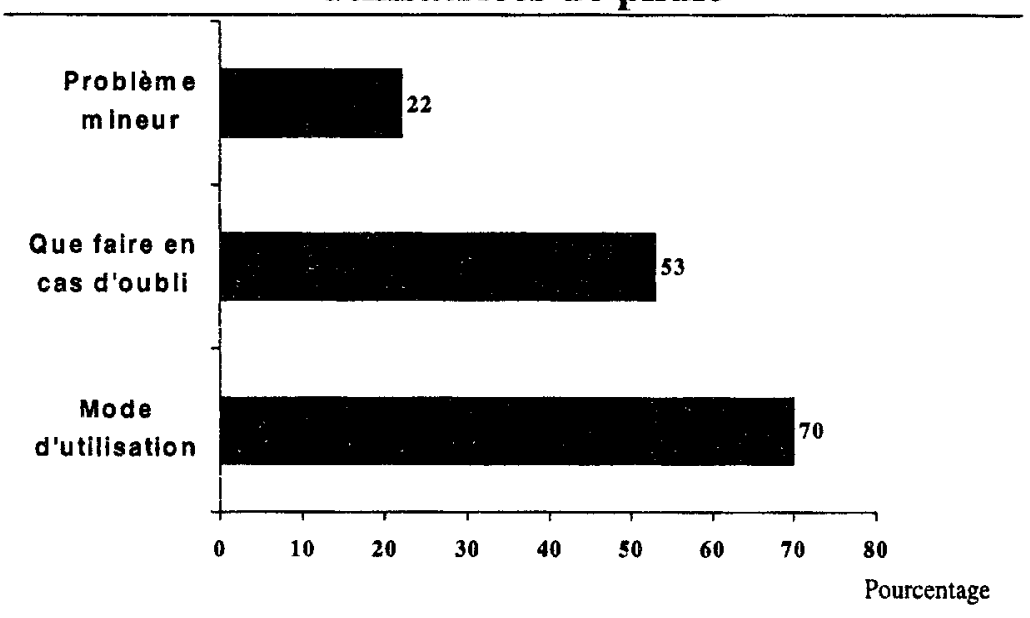

Source: Interview cliente PF
PF $\quad(4 \%)$. En outre l'information sur la prévention des MST/SIDA par l'usage du préservatif est faiblement abordée au cours des consultations de PF: $33 \%$ des nouvelles clientes et $8 \%$ des anciennes clientes respectivement.

\subsection{Informations retenues par les clientes.}

L'interview des clientes de PF à la sortie de leur consultation a permis de constater que cellesci retiennent très peu l'information qui leur est

donnée sur la méthode acceptée. En général, les clientes ont un bon niveau de connaissance du mode d'utilisation de leur méthode. Pour la pilule, $70 \%$ d'entre elles savent quand il faut commencer à la prendre, $95 \%$ qu'il faut la prendre tous les jours. Cependant $53 \%$ savent ce qu'il faut faire en cas d'oubli. Les clientes ont de faibles connaissances sur les problèmes mineurs et encore moins sur les problèmes majeurs. Les clientes de pilule savent qu'elles pourraient avoir des nausées $(31 \%)$, des migraines $(28 \%)$, des saignements $(15 \%)$ et une prise de poids $(7 \%)$. Concernant les signes d'alarme nécessitant leur retour au PPS, elles ont cité les douleurs aigües dans la poitrine ou essouflement (13\%) et les maux de tête (22\%).

Les utilisatrices de DIU ont surtout cité les saignements minimes (38\%), les infections et douleurs dans le bas ventre et les pertes blanches (13\%). Les utilisatrices d'injectable ont cité les légers maux de tête (22\%), les nausées (12\%), les saignements irréguliers (27\%) la prise de poids $(8 \%)$ et l'aménorrhée $(30 \%)$. 


\section{Compétence technique des prestataires}

L'observation de la prise en charge clinique de la femme permet d'apprécier la compétence technique des prestataires. Cette compétence se rapporte principalement au respect des protocoles recommandés par le Ministère de la Santé pour les consultations prénatales, les consultations MST/SIDA, les consultations PF et les consultations post natales.

\subsection{Procédures jugées nécessaires par les prestataires de PF}

\section{Graphique No 20}

Procédures jugées nécessaires par les prestataires avant d'offir la pilule et le DIU

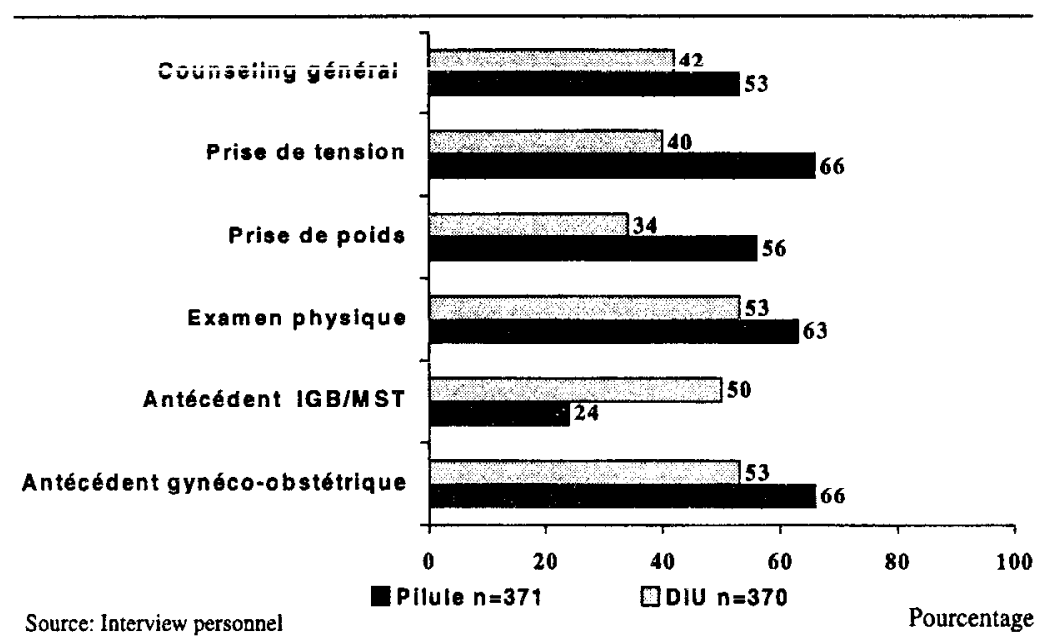

Il apparaît à partir de l'interview du personnel que tous les prestataires de service n'ont pas cité les principales procédures

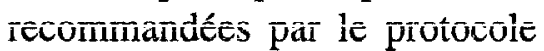
de prise en charge. Aux clientes qui se présentent pour la première fois, la prise de tension, la prise de poids, les antécédents gynécologiques et l'examen physique sont les préoccupations d'environ trois quart des prestataires. Le counseling est moins cité. Il apparaît que les prestataires respectent dans une plus grande proportion les protocoles lorsqu'il s'agit de la pilule comparé au DIU. Pour ce dernier, ce sont les antécédents sur les infections génitales basses et les MST que les prestataires recherchent plus souvent.

\section{Graphique No 21}

Procedures médicales accomplies durant l'examen pelvien

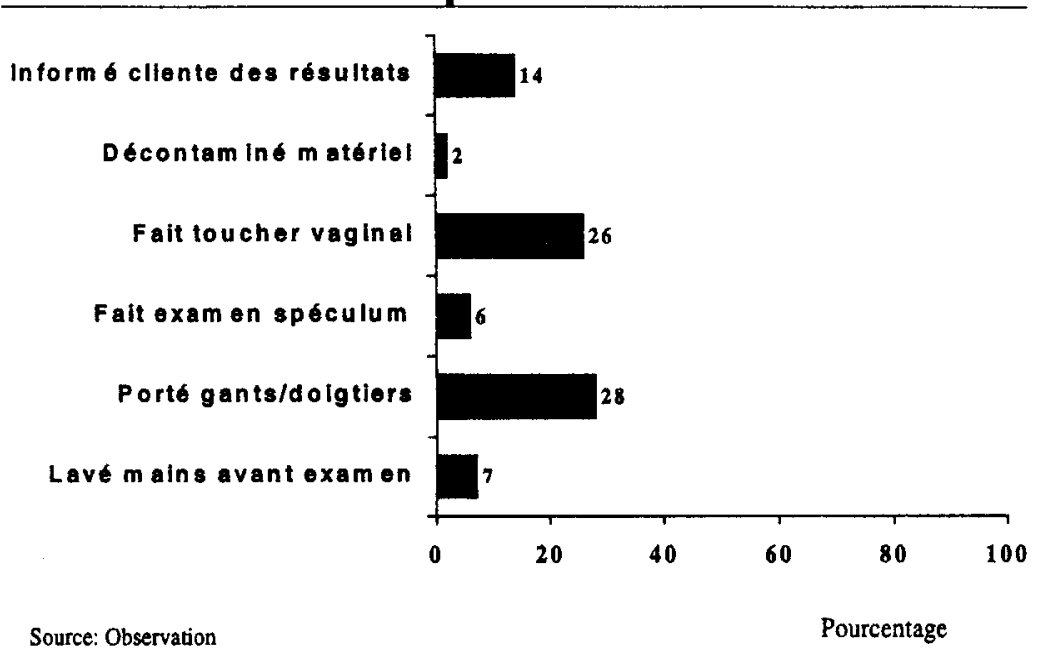

En face d'une cliente désirant une méthode et n'ayant pas ses menstruations, la moitié des prestataires lui disent de revenir lorsqu'elle aura ses règles ou fournissent le préservatif et demandent de revenir au moment des règles. Il y a $4 \%$ qui ont déclaré provoquer les menstruations et $6 \%$ qui fournissent la méthode en plus des préservatifs.

L'observation des consultations révèle que dans la pratique, le 
poids et la tension de la femme ont été pris par $84 \%$ des prestataires. L'examen mammaire et la vérification de l'anémie est faite par la moitié d'entre eux. La recherche de leucchorées inhabituelles, de douleurs au bas-ventre et de saignements vaginaux est moins systématique. Et $30 \%$ des nouvelles clientes ont eu un examen pelvien. Durant l'examen pelvien les conditions d'aseptie ne sont pas rigoureusement observées. En effet pour trop peu de clientes les prestataires se sont lavés les mains avant l'examen et ils ont porté des gants. Pour ceux qui ont porté des gants l'étude s'intéressait à leur état. Cependant la plupart des observateurs n'ont pas pu déterminer s'ils étaient stériles ou non. On a reporté que $18 \%$ des gants ou doigtiers étaient propres mais pas stérilisés. Finalement, très peu de clientes sont informées des résultats de l'examen physique.

La prise en charge des clientes ayant eu des problèmes avec les méthodes reste un élèment essentiel pour la continuité. Les effets secondaires étant les problèmes les plus souvent cités (55\%), il apparaît qu'en général les prestataires en discutent surtout avec la cliente et la rassure. Dans seuiement 1 cas, le prestataire a suggéré un changement de méthode.

\subsection{Procédures médicales des consultations prénatales.}

Lors des premières visites ainsi que des visites subséquentes, la hauteur utérine est prise pour presque toutes les femmes ainsi que la prise de la tension. Cependant la prise de poids est surtout effectuée pour les premières visites prénatales. L'examen des muqueuses est pratiqué dans une moindre mesure. La recherche d'oedème chez les femmes a été observée dans seulement le tiers

\section{Graphique $\mathbf{N}^{0} 22$}

Procédures médicales accomplies lors des consultations prénatales

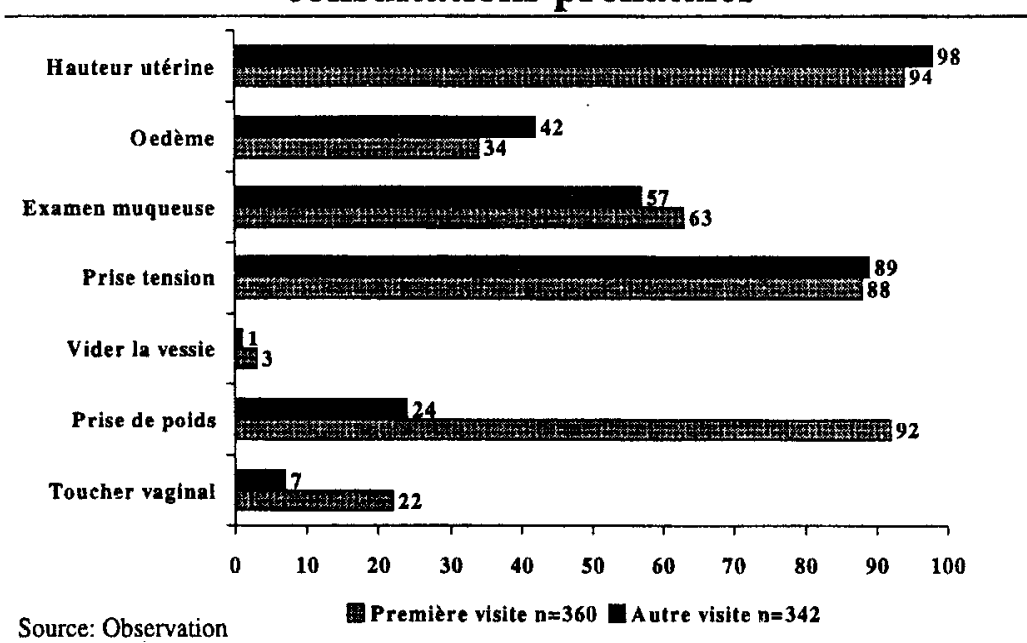

des consultations. I n'est pratiquement jamais demandé à la patiente de vider la vessie. Pour les nouvelles consultantes, c'est la recherche d'albumine et de sucre dans les urines qui est le test le plus pratiqué. Les autres test sanguins, d'Emmel, d'hemoglobine sont peu pratiqués.

Les prescriptions faites par les prestataires sont le fer $(79 \%)$, la chloroquine $(91 \%)$ et le VAT $(84 \%)$.

\subsection{Aptitude du personnel à prendre en charge les services de MST/SIDA}

De l'observation des consultations, il apparaît que les trois quart des prestataires recherchent surtout les leucchorées ou les ulcérations. Les examens gynécologiques ou urologiques et la recherche de fièvre sont pratiqués par un peu plus de la moitié des prestataires. Cependant la 
recherche de ganglions, de lésion de la peau ou de lésion de la bouche a été pratiquée par peu de prestataires.

Graphique No 23

Procédures médicales accomplies lors des consultations MST/SIDA

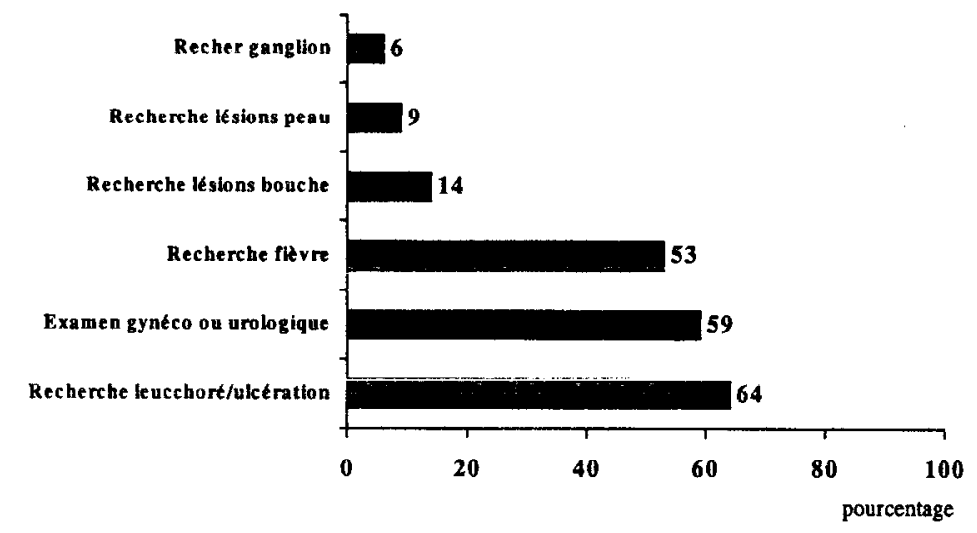

Lors des interviews avec les prestataires, des questions sur la prise en charge des patient(e)s atteintes de MST ou de SIDA ont également été posées. A la question "que faire si un(e) patient(e) a une MST" $76 \%$ des prestataires ont répondu qu'ils allaient traiter, $40 \%$ qu'ils allaient demander à voir le partenaire, $39 \%$ qu'ils allaient faire du counseling. La mềme question a été posée à propos du VIH/SIDA. On note que la première attitude d'un prestataire face à un(e)

patient(e) semblant être atteinte par le VIH/SIDA c'est de chercher à confirmer le diagnostic en reférant pour des tests. Enfin, 39\% ont déclaré qu'ils feraient du counseling et $37 \%$ qu'ils donneraient des préservatifs.

\subsection{Procédures lors des consultations postnatales}

L'observation des 67 femmes venues pour consultation postnatales révèle que l'intérêt a surtout porté sur les informations relatives à l'accouchement et l'examen de l'abdomen. Dans une moindre mesure il y a eu un examen de la muqueuse et des conjonctives et un examen de la vulve et du périné. La prise du pouls et de la température a été fait avec le quart des clientes. Les données montrent également que $34 \%$ des femmes ont été reférées en CPC.

\section{Mécanismes pour encourager la continuité}

Au cours de l'enquête, l'on a constaté que la majorité des clientes avaient leur méthode le jour de la visite: respectivement $76 \%$ et $84 \%$ des clientes nouvelles et anciennes.

En général, la plupart des nouvelles clientes (55\%) qui n'ont pas pu obtenir leur méthode le jour de la visite devaient attendre de voir leurs règles. Les autres raisons étaient plutôt la rupture de stocks (21\%) et la pose de DIU ou une ligature de trompes (15\%) nécessitant un retour. Aucune 
nouvelle cliente n'a été réferée vers une autre structure pour recevoir la méthode contraceptive voulue.

La date de la prochaine visite a été indiquée à presque toutes les clientes de PF (98\% des nouvelles et $93 \%$ des anciennes). Cependant il faut préciser que seulement $88 \%$ des nouvelles clientes ont reçu un papier ou un carnet portant mention de la date de rendez-vous. En outre, pour le suivi ou pour le réapprovisionnement, les agents de santé ont demandé aux clientes de revenir dans le même PPS (plus de $96 \%$ des clientes).

Aux autres clientes de SR également, les dates de la prochaine visite ont été communiquées: A $93 \%$ des clientes de prénatales, à $54 \%$ des clientes postnatales et à $48 \%$ des client(e)s de MST/SIDA.

Pour les nouvelles acceptrices de PF, un cycle de pilule est généralement donné. Ce qui est conforme aux normes et procédures. Ces normes ne sont pas toujours respectées puisque $14 \%$ des nouvelles clientes ont reçu plus d'un cycle de pilule. L'interview de la cliente PF montre que pour la visite de controle qui doit s'effectuer normalement un mois après la pose du DIU. A seulement $25 \%$ des nouvelles utilisatrices de DIU, on a dit de revenir à cette periode.

\section{Organisation des services}

L'un des objectifs primordiaux du Ministère de la Santé est d'intégrer la planification familiale dans les soins de santé primaire. Les centres de santé sont les PPS qui doivent en priorité fournir les services de SR. A ce jour, 335 centres de santé sont fonctionnels. La PF est déjà intégrée dans environ $56 \%$ de ces centres. L'intégration des services a porté sur l'examen des différents types d'activités menées dans les PPS, sur le nombre de jours dans la semaine et le nombre d'heures de fonctionnement par jour.

Les données recueillies à travers le questionnaire inventaire permettent de constater que la PF est offerte avec les consultations prénatales et postnatales, les services de vaccination, les accouchements, les services curatifs et les causeries éducatives en matière de santé dans bon nombre de PPS. Tous ces services sont offerts pendant six jours par semaine dans plus d'un PPS sur deux. La durée de fonctionnement varie selon le PPS. Dans environ 14\% des PPS la durée de fonctionnalité a été estimée à moins de 3 heures par jour et dans 19\% à 4 heures par jour.

L'observation de l'interaction prestataires-clientes $\mathrm{PF}$ révèle que pendant la consultation $\mathrm{PF}$, les autres problèmes de santé ne sont presque jamais abordés. Quand cela arrive, les prestataires parlent du VIH/SIDA (11\% des observations), des MST (15\%), de l'avortement (7\%) et de l'éducation sexuelle (7\%). Un autre indicateur du niveau d'intégration des services est la proportion de clientes SMI qui ont vu ou entendu quelque chose en rapport avec la PF dans le PPS le jour de leur visite. En effet, seulement $20 \%$ des clientes de SMI ont déclaré avoir vu ou entendu quelque chose sur la PF. Parmi elles, une cliente sur deux a vu une affiche sur la PF. Celles qui ont vu des échantillons de contraceptifs, qui ont assisté à des causeries éducatives ou qui ont entendu quelque chose sur la PF représentent respectivement $36 \%, 25 \%$ et $16 \%$ de ces clientes. Très peu d'entre elles $(5 \%)$ ont vu un dépliant, une boîte à image ou une brochure sur la PF. 


\section{Conclusion}

La qualité des services dans les structures de santé comporte des forces et des faiblesses. Du point de vue satisfaction, on peut voir qu'une majorité de clientes ont déclaré être satisfaites et avoir eu un temps d'attente raisonnable. Cependant une majorité des clientes de SMI se sont aussi plaintes du temps de consultation trop court. Les informations échangées entre les prestataires et les clientes sont insuffisantes. Ceci est confirmé par la faible connaissance des clientes interrogées à la sortie des consultations.

Les protocoles de prise en charge clinique ne sont pas entièrement respectés par les prestataires. Peu d'attention est donnée aux règles d'hygiène pour la prévention des infections. Cependant les mécanismes d'encouragement de la continuité des différentes composantes de la SR sont assez respectés.

\section{Recommandations sur la qualité des soins des femmes}

1. Faire une large diffusion à tous les niveaux de la pyramide sanitaire des normes et procédures en SR.

2. Faire des supervisions formatives en assistant le prestataire dans la prise en charge des client(e)s.

3. Insister au cours des supervisions sur le respect des règles d'hygiène nécessaires à la prévention des infections.

4. Elargir la formation en santé de la reproduction aux agents techniques de Santé. 


\section{E- QUALITE DES SOINS POUR LA SURVIE DE L'ENFANT}

Une composante sur la survie de l'enfant a été introduite dans l'étude, avec l'assistance technique du projet BASICS. Cette composante avait pour objectif d'évaluer la qualité des soins offerte aux enfants malades de moins de 5 ans à partir de l'observation de leur prise en charge, de l'interview des mères ou accompagnant(e)s à la sortie des consultations et de l'interview des agents de santé.

\section{Motifs de la visite et recherche des symptômes}

\subsection{Motif de la visite}

Les motifs de consultation des enfants malades qui devraient faire l'objet d'une observation sont la toux ou le rhume, les infections respiratoires aïgues, la diarrhée, la fièvre/paludisme, l'anémie ou la malnutrition. Un enfant malade pouvait venir consulter nour phlusieurs motifs In est anpparn que le motif de consultation le plus fréquemment évoqué est la fièvre qui touche $90 \%$ des enfants de l'échantillon. Les autres symptômes les plus souvent associés à ce premier motif sont la toux, le rhume ou les difficultés respiratoires (52\%), la diarrhée (32\%), l'anémie, la malnutrition ou les autres raisons $(62 \%)$. Il faut préciser que la durée médiane de consultation la plus faible enregistrée au cours de cette étude est celle consacrée à un enfant malade soit $8 \mathrm{mn}$.

\section{Graphique $\mathbf{N}^{0} 25$}

Répartition des enfants selon le nombre de motifs de consultation.

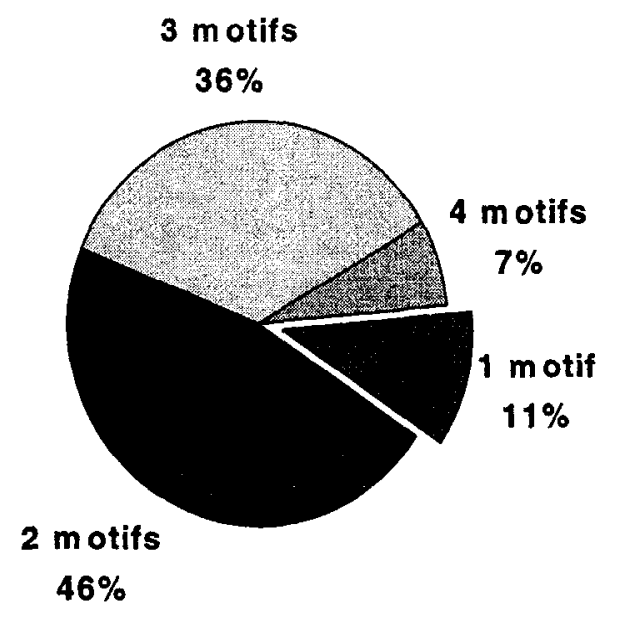

Les résultats montrent que seulement $11 \%$ des enfants malades avaient un seul motif de consultation. $\quad \mathrm{La}$ distribution des motifs de consultation selon l'âge montre d'ailleurs que dans la tranche d'âge de 0 à 11 mois, $36 \%$ des enfants préscntaient quatre motifs de consultation contre $32 \%$ pour la tranche de 36 mois à 59 mois. Cette situation serait en relation avec la période de l'enquête qui a coincidé avec la saison

des pluies.

Les données montrent que $32 \%$ des motifs fièvre sont enregistrés au niveau de la région forestière contre $12 \%$ pour la région de Conakry. Plus d'un enfant sur deux présentant ce motif appartient à la zone rurale. Plus de $37 \%$ des cas de fièvre sont enregistrés au niveau de la tranche d'âge de 0 à 11 mois. 
Le motif « toux » est noté chez $31 \%$ de la zone forestière contre $14 \%$ pour la région de Conakry. Un enfant sur deux de la zone rurale en souffrait. Au niveau de l'âge, on trouve $42 \%$ chez les moins d'un an contre $16 \%$ chez ceux âgés de 36 à 59 mois.

Le motif «diarrhée» est présent chez $36 \%$ des enfants de la zone forestière contre $14 \%$ pour la région de Conakry. Plus d 'un enfant sur deux avait ce symptôme dans la zone rurale. Sur trois cent cinq enfants qui souffraient de diarrhée, $38 \%$ étaient dans la tranche d'âge de 0 à 11 mois.

La répartition du nombre de motifs de consultation par zone permet de constater que près de $69 \%$ des enfants observés dans la zone péri-urbaine présentaient au moins deux motifs contre $46 \%$ pour la zone rurale et $47 \%$ pour la zone urbaine. Dans toutes les trois zones, plus de trente cinq (35\%) pour cent des enfants présentaient au moins trois symptômes. Dans la zone urbaine 51\% avaient quatre motifs de consultation alors que ce taux est de $6 \%$ pour la zone rurale.

\subsection{Recherche des principaux symptômes}

\section{Graphique No 26}

Proportion d'enfants pour lesquels les symptômes ont été recherchés

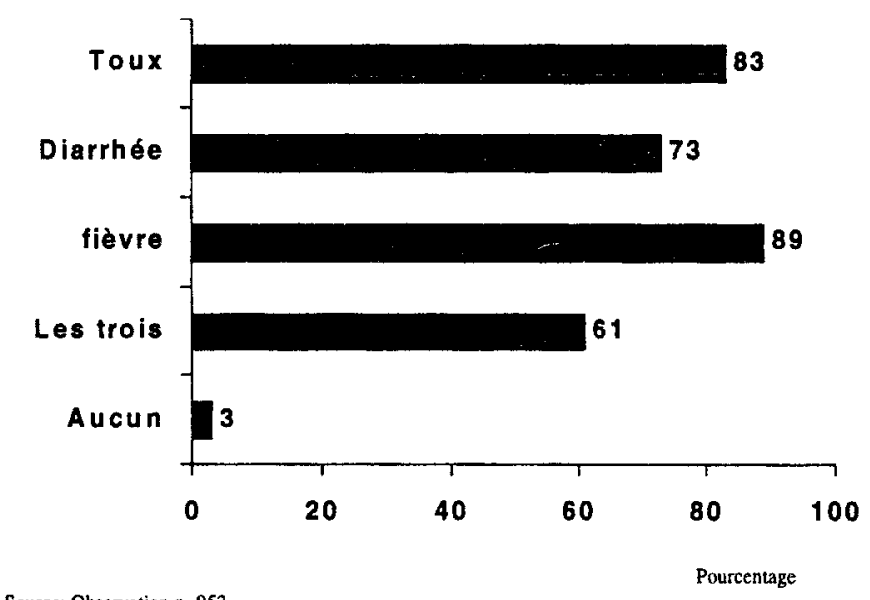

Source: Observation $n=953$
Dans les normes de prise en charge intégrée des maladies de l'enfant, il est attendu que l'agent de santé évalue systématiquement tout enfant qui vient à la consultation pour les trois principaux symptômes, à savoir la fièvre, la toux et la diarrhée, et ce, quel que soit le motif de la visite.

Sur les 954 enfants vus par un agent de santé en service curatif, $89 \%$ ont été systématiquement

évalués pour la fièvre, $83 \%$ pour la toux et $73 \%$ pour la diarrhée. Au total, 6 enfants sur 10 ont été évalués pour les trois principaux symptômes et environ un enfant sur quatre pour deux principaux symptômes.

\subsection{Recherche des signes de danger}

Quatre signes de dangers sont à rechercher chez les enfants malades :une incapacité de boirc ou de s'alimenter, le fait que l'enfant vomit tout ce qu'il prend, une léthargie et des convulsions.

Au cours des consultations, le personnel de santé n'a recherché aucun de ces quatre signes chez $42 \%$ des enfants malades. Les enfants pour lesquels tous les signes de danger sont recherchés représentent seulement $1 \%$. 
Par ailleurs, l'étude a montré que la situation est nettement meilleure à Conakry où les prestataires ont recherché les quatre signes chez $19 \%$ des enfants malades. Dans les autres régions, le personnel de santé n'a presque pas cherché les signes de danger chez les enfants malades. A Boké et Labé, par exemple, le personnel n'a cherché ces signes que chez respectivement $1 \%$ et $6 \%$ des enfants malades. Environ $12 \%$ des médecins et des infirmiers ont recherché trois signes de danger contre seulement $4 \%$ des techniciens de santé.

\section{2- Evaluation de l'enfant par le personnel de santé}

C'est 44\% des enfants malades qui ont été examinés par les agents techniques de santé (ATS). Les infirmiers sont, après les ATS, les prestataires qui font le plus de consultations d'enfants malades (29\%). Les autres enfants ont été pris en charge par des médecins (23\%) et d'autre type de personnel $(4 \%)$. Les sages femmes ne sont pratiquement pas impliquées dans la prise en charge des maladies de l'enfant $(1 \%)$.

Chaque symptôme présenté par l'enfant doit faire l'objet d'une évaluation systématique qui passe au crible tous les signes clés des maladies susceptibles d'engendrer ce symptôme. Des éléments d'identification de la toux, de la diarrhée et de la fièvre doivent permettre aux agents de santé de poser leur diagnostique.

\subsection{Evaluation de la toux et des difficultés respiratoires}

\section{Graphique No 27}

Distribution des enfants selon les éléments d'évaluation de la toux recherchés par l'agent

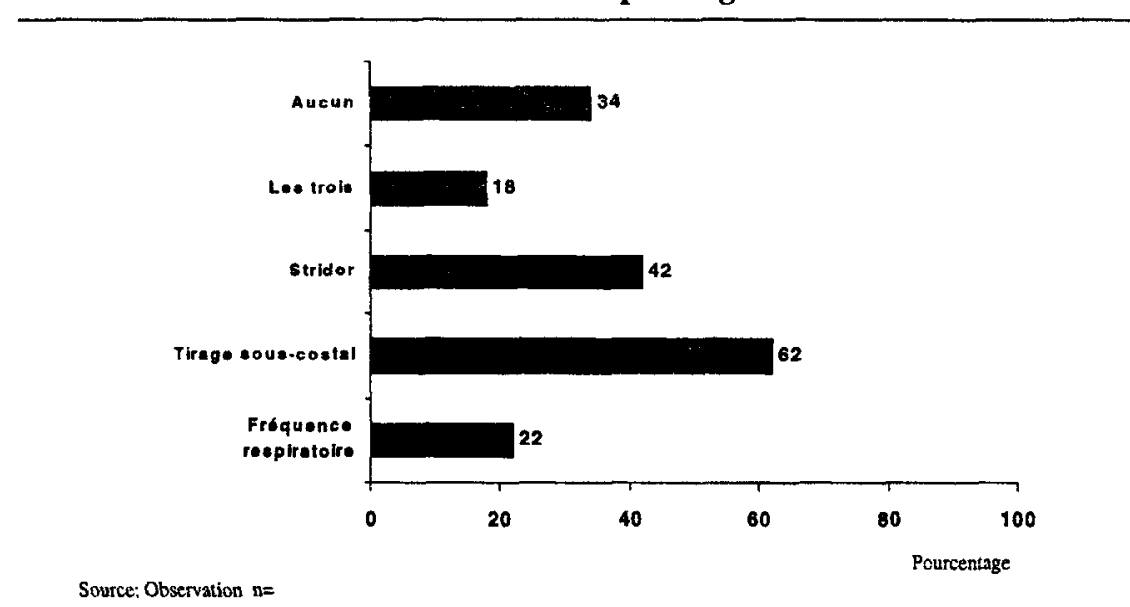

Selon les normes et procédures, pour correctement évaluer la toux ou les difficultés respiratoires chez l'enfant il faut compter les fréquences respiratoires de l'enfant, écouter la poitrine de l'enfant, rechercher un stridor ou une respiration sifflante.

Les données permettent de constater que chez $31 \%$ d'enfants malades, les prestataires ont accompli

un geste sur les trois. Les 3 gestes nécessaires pour évaluer correctement la toux ou les difficultés respiratoires ont été posés par les prestataires chez $18 \%$ d'enfants malades. Trois signes ont été recherché par au moins $38 \%$ des infirmiers d'Etat et environ $8 \%$ des médecins. Quant aux sages-femmes, dans 50\% des cas elles ont recherché deux signes sur les quatre. 


\subsection{Evaluation de la diarrhée}

Pour évaluer la diarrhée chez l'enfant, le personnel de santé doit effectuer les 4 tâches suivantes : vérifier la présence de sang dans les selles, vérifier si l'enfant a soif et s'il est capable de boire, pincer la peau pour la recherche de pli cutané, examiner l'enfoncement des yeux.

Les prestataires ont accompli à la fois les 4 tâches ci-dessus citées dans l'évaluation de la diarrhée chez seulement $9 \%$ des

Graphique No 28

Distribution d'enfants selon les éléments d'évaluation de la diarrhée recherchés par l'agent

enfants malades. Les quatre signes ont été recherchés par $11 \%$ des infirmiers d'Etat, $8 \%$ des agents techniques de santé et seulement $3 \%$ des médecins. L'enfoncement des yeux est recherché pour $59 \%$ des enfants se plaignant de diarrhée, le pli cutanée pour $55 \%$ des enfants. On constate que la présence de la soif n'est recherché que chez $10 \%$ des enfants. Pour $19 \%$ des enfants ayant une plainte de diarrhée, aucun des signes n'a été recherché.

\subsection{Evaluation de la fièvre}

Graphique No 29

Distribution d'enfants selon les éléments d'évaluation de la fièvre recherchés par l'agent

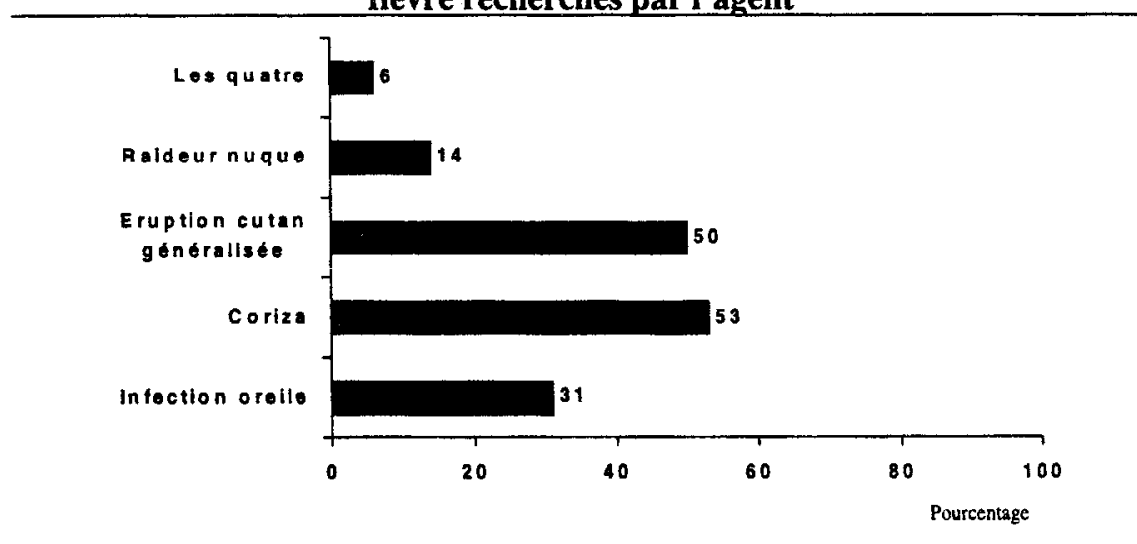

Le personnel de santé pose un certain nombre de gestes pour vérifier si un enfant a de la fièvre. Parmi ces gestes, quatre ont ćté retenus commc tâches importantes dans l'évaluation de la fièvre chez l'enfant. En effet, ils doivent vérifier si l'enfant a une raideur de la nuque, une éruption généralisée, un coryza et la présence d'une infection de l'oreille.

On constate que les prestataires n'ont accompli toutes les 4 tâches que chez $6 \%$ d'enfants malades. Les enfants malades pour lesquels, aucune de ces tâches n'a été accomplie représentent 
$24 \%$. Les quatre signes ont été recherchés dans $9 \%$ des cas pris en charge par un infirmier d'Etat contre $5 \%$ pour les agents techniques de santé et seulement $3 \%$ des cas pris en charge par un médecin.

\subsection{Evaluation de l'état nutritionnel et de l'alimentation}

Graphique No 30

Distribution des enfants selon les éléments d'évaluation de l'état nutritionnel recherchés par l'agent

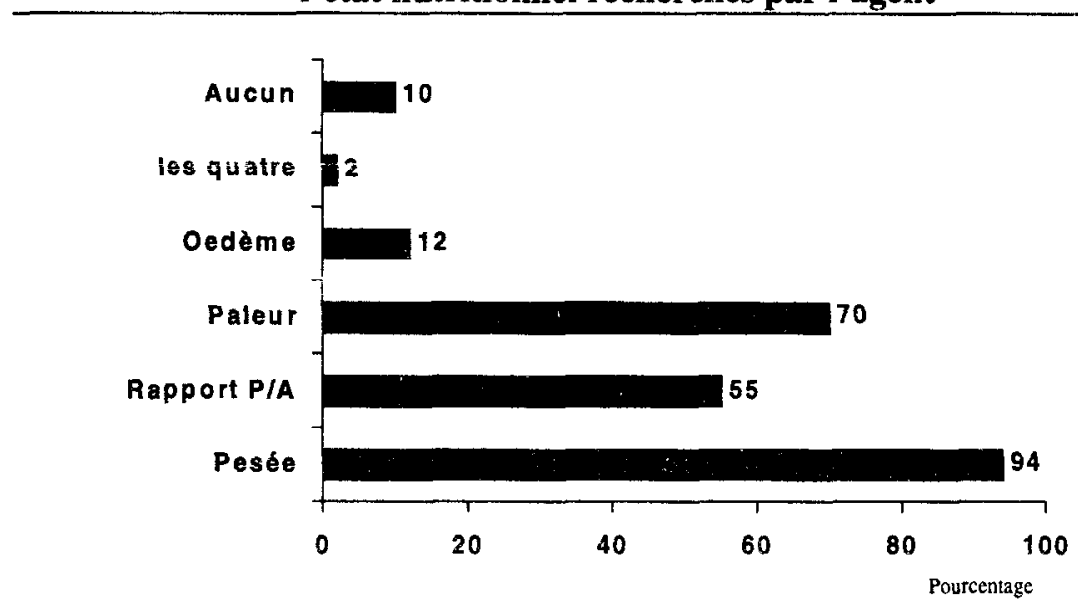

Source: Observation $n=954$
Un intérêt particulier a été porté sur la malnutrition et l'alimentation de l'enfant. L'état nutritionnel de l'enfant devrait être systématiquement évalué lors des consultations curatives ainsi que l'alimentation durant la maladie. L'évaluation de l'état nutritionnel comporte la pesée de l'enfant et le traçage de la courbe de rapport poids/âge, la recherche des oedèmes des membres inférieurs et la pâleur des paumes.

Au total $2 \%$ seulement des 954 observés ont été correctement évalués pour l'état nutritionnel. Tandis que $94 \%$ ont été pesés, 55\% seulement ont eu le traçage de la courbe de rapport poids/âge. La pâleur des paumes a été recherchée pour environ 7 enfants sur 10.

Pour $10 \%$ des enfants aucune de ces tâches d'évaluation n'a été effectuée.

Les résultats font ressortir que $60 \%$ des enfants pris en charge par une sage-femme ont eu au moins trois tâches d'évaluation de l'état nutritionnelle

Les performances en ce qui concerne les tâches d'évaluation de l'alimentation de l'enfant font apparaître que $2 \%$ seulement des enfants ont été évalués pour leur ration alimentaire et $5 \%$ pour le nombre de repas qu'ils prennent par jour. L'agent de santé a demandé si l'enfant avait un complément alimentaire dans $18 \%$ des cas. Dans $96 \%$ des cas l'agent de santé a cherché à savoir si l'enfant était sous allaitement exclusif. Pour plus de 7 enfants malades sur 10, aucune des questions n'a été demandée. Une seule question sur les cinq prévues a été posée pour $15 \%$ d'enfants malades. Les sages-femmes et les agents techniques de santé ont effectué au moins quatre tâches d'évaluation de l'alimentation dans $80 \%$ des cas contre $73 \%$ pour les infirmiers d 'Etat et $71 \%$ pour les médecins. 


\subsection{Evaluation du statut vaccinal}

Graphique No 31

Pourcentage d'enfants pour lesquels l'agent a demandé le

carnet, vérifié l'état vaccinal, et entrepris une action

L'évaluation du statut vaccinal comporte la vérification de la disponibilité du carnet de vaccination, l'état de complétude des vaccinations du programme élargi de vaccination et la mise à jour au cas échéant.

L'agent de santé a demandé à la mère/accompagnant si elle/il avait apporté le carnet de vaccination seulement dans $38 \%$ des cas. L'état de complétude des vaccinations de l'enfant malade a été vérifié dans $18 \%$ des cas. L'enfant a été référé ou a reçu une mise à jour de son état vaccinal dans $3 \%$ des cas seulement.

Parmi les cas pris en charge, la disponibilité du carnet de santé de l'enfant et de la mère a été simultanément vérifiée dans $10 \%$ des consultations faites par un agent technique de santé et moins de $5 \%$ des cas pris en charge par un médecin et un infirmier d'Etat.

Il faut préciser que pour $62 \%$ des enfants malades, l'agent de santé n'a pas demandé le carnet. II n'est alors pas surprenant de constater que le statut vaccinal n'a été évalué que pour $49 \%$ des enfants malades. Sur 954 mères ou accompagnants de l'enfant malade, le carnet de santé a été demandé dans $7 \%$ seulement des cas et le statut vaccinal de la mère ou accompagnant, dans $2 \%$ des cas.

En ce qui concerne la référence de la mère dont le statut vaccinal a été évalué pour une action de mise à jour de ses vaccinations, cette tâche a été effectuée dans $2 \%$ des cas seulement.

La vérification de l'état vaccinal de la mère ou de tout autre accompagnant de l'enfant malade notamment les femmes adolescentes ou non encore ménauposées, devrait être systématique pour accroître les chances de réduire les occasions manquées. 


\section{Conseils sur l'administration des médicaments et communication interpersonnelle}

Pour que l'enfant recouvre rapidement sa santé, il faut un suivi régulier à domicile ou encore une prise en charge de l'administration des médicaments par les parents. Les agents de santé devraient donner des instructions claires aux mères ou aux accompagnat(e)s des enfants malades et vérifier que ces instructions sont bien comprises. Les résultats montrent qu'en général, les prestataires donnent des conseils au sujet de l'administration des médicaments oraux puisque $86 \%$ des mères ont déclaré avoir reçu de tels conseils. Malheureusement, ils ne vérifient que très peu si les mères ont compris les conseils donnés. En effet cette vérification a été effectuée pour seulement $3 \%$ des cas. Les conseils qu'il est demandé aux prestataires de fournir à la mère portent sur les points suivants :

Graphique $\mathbf{N}^{\circ} 32$

Distribution des mères selon les éléments de conseil reçus sur la prise en charge en général à domicile

- Des instructions sur l'alimentation, les liquides, l'administration des médicaments ;

- Les signes alarmants nécessitant un retour à la formation sanitaire (enfant ne mange pas, ou ne boit pas, ou vomit tout ce qu'il prend et/ou ne se porte pas mieux).

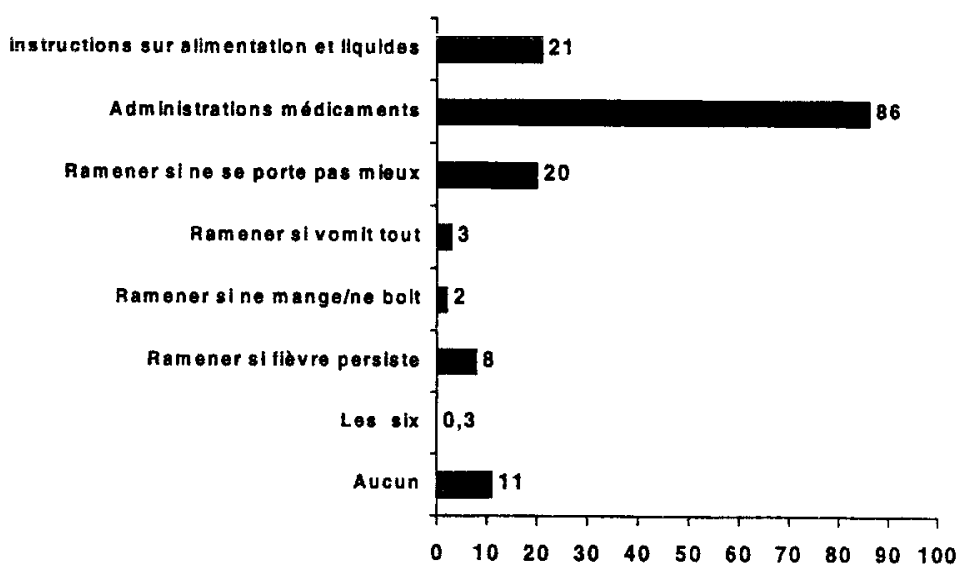

Il apparaît que les mères ne sont presque jamais correctement conseillées pour la prise en charge à domicile. Les conseils sur l'alimentation de l'enfant malade ont été donnés dans $21 \%$ des cas. Les conseils sur les signes d'alarme qui doivent pousser la mère ou l'accompagnant à ramener l'enfant à la formation sanitaire ne sont donnés que dans une proportion allant de $2 \%$ à $8 \%$. Près de $11 \%$ des mères n'ont reçu aucun de ces conseils. La distribution des mères selon les conseils reçus fait ressortir que $0,3 \%$ des mères ont reçu tous les six (6) conseils et la majorité des mères $(58 \%)$ ont reçu seulement un conseil sur les six.

Communication efficace. La communication interpersonnelle occupe une place importante dans la qualité de la prise en charge de l'enfant malade. Les agents de santé devraient dans tous les cas créer les conditions d'une communication efficacc. Ils doivent donc poser des questions ouvertes, demander si la mère/accompagnant(e) a des questions à poser, lui dire quand revenir pour la visite de suivi et la féliciter pour les bonnes pratiques.

Avec $79 \%$ des mères ou accompagnant(e)s aucune de ces pratiques n'a été faite. Une condition sur les trois précitées a été remplie pour seulement $19 \%$ des mères. Pour $9 \%$ des mères, deux conditions ont été remplies sur les trois. Aucune mère n'a eu une communication efficace avec les agents de santé c'est-à-dire toutes les trois conditions n'ont été remplies pour aucune mère. Les agents de santé ont surtout donné des instructions pour le rendez-vous de suivi (19\%), alors 
que la vérification de la compréhension n'a été faite que pour 3\% des cas. La distribution des mères selon le nombre de conseils reçus et le type de prestataire fait ressortir que $18 \%$ des agents techniques de santé ont donné au moins trois conseils contre $12 \%$ des infirmiers d 'Etat et seulcment $6 \%$ des médecins.

\section{Connaissances des mères en santé infantile}

Connaissance de la chloroquine et du paracetamol. L'interview des mères/accompagnant(e)s à la sortie de la consultation a permis de mesurer certains indicateurs relatifs à leur connaissance et pratique. En général, les mères ont une bonne connaissance de l'administration de la chloroquine. Presque toutes (99\%) connaissent la dose par prise et le nombre de prises par jour (90\%). Cependant, la proportion de mères qui connaissent la durée exacte du traitement pour la chloroquine est plus basse $(77 \%)$.

La connaissance du paracétamol est moins bonne. Seulement $8 \%$ des mères/accompagnant(e)s connaissent la dose correcte par prise. Elle est de $16 \%$ en Moyenne Guinée et de $0.1 \%$ en Basse Guinée. Le nombre de prise par jour et la durée du traitement est connue de $8 \%$ des personnes ayant reçu la prescription le jour de la visite.

Maîtrise de la dilution des sachets de SRO. La prévention de la déshydratation chez l'enfant atteint de diarrhée par le SRO est connue de $74 \%$ des mères/accompagnant(e)s qui ont été interviewé(e)s. Les autres mères (26\%) ont montré des lacunes dans l'information qu'elle possédaient.

Connaissance du paquet d'activité préventives. On note une très faible connaissance des mères sur la prévention du paludisme. Dix pour cent des mères ont cité la moustiquaire comme mesure préventive et seulement $1 \%$ d'entre elles ont cité la moustiquaire imprégnée. Quant à la chloroquine seules $17 \%$ des mères savent la nécessité d'en prendre durant la grossesse pour la prévention du paludisme.

\section{Connaissance des agents de santé sur la santé infantile}

Connaissances des doses de Vitamine A. L'interview des agents de santé montre que les prestataires de service ne maîtrisent pas les doses nécessaires de Vitamine A dans le traitement de la rougeole. Moins de $2 \%$ des agents connaissent les doses pour les enfants de moins de 6 mois et pour ceux de 6 à 11 mois. Pour les enfants âgés de 12 mois et plus et pour la femme après l'accouchement, les doses ne sont connues par aucun agent.

Connaissance des groupes cibles du PEV. Sur 582 agents interrogés, 59\% ont cité les groupes cibles du vaccin antitétanique, $25 \%$ celui du BCG/DTCP, $22 \%$ celui du vaccin contre la rougeole. Moins de 7\% des agents ont cité toutes les cibles des principales vaccinations du PEV. 


\section{Conclusion}

De l'ensemble des résultats constatés, les tendances suivantes se dégagent.

II existe une opportunité pour promouvoir l'introduction de la prise en charge intégrée des maladies de l'enfant, mise en évidence par le fait qu'une majorité d'enfant ont été systématiquement évalués pour les trois principaux symptômes à savoir la fièvre, la toux et la diarrhée.

La qualité des prestations reçues par les enfants malades dans les points de prestations sanitaires est très basse comparée aux normes de la Prise en Charge Intégrée des Maladies de l'Enfant (PCIME).

La majorité des mères maîtrisent l'administration du SRO alors que très peu d'entre elles connaissent les moyens de prévenir le paludisme.

Les agents de santé interviewés, en majorité des ATS, ne maîtrisent pas le Programme Elargi de Vaccination et l'administration de la Vitamine A.

\section{Recommandations pour la Survie de l'enfant}

1. Profiter des opportunités damélioration identifiées par cette étude pour accélérer Pintroduction de la strategie de Prise en Charge Integrée des Maladies de PEnfant (PCIMI) dans les P DS.

2. Faire un plaidoyer auprès de tous les intervenants du secteur de la santé en vue d'elaborer un plan d'action intégré en faveur de l enfant.

3. Faire le plaidoyer aupres des bailleurs de fonds en vie d bobtenit les moyens de renforcer Iapput au Ministere de la Sante de Guinee.

\section{Ameliorer la formation du personiel de sante.}

5. Augmenter le nombre de personnel qualite pour la prise en charge de la survie de Tenfant.

\section{Assure le suivi et levaluation des formations.}

7. Tire une large diffusion des no rmes et protocoles de las survie de 1 cenfant. 


\section{RÉFÉRENCES BIBLIOGRAPHIQUES}

Bruce Judith and Andruth Jain. Fundamental Elements of the Quality of Care. A simple Framework. Population Council New York. 1991.

Diop, B. M., Geslin, C., Kelly, P. Enquête auprès des établissements de santé sur la prise en charge de l'enfant malade, Districts sanitaires de Say et de Boboye, du 18 au 20 novembre 1996, République du Niger, Report, BASICS.

Durgavich, J. \& Mutombo Wa Mutombo, Rapport de Mission (Draft). Détermination des besoins en assistance technique au bénéfice de la Mission de l'USAID, du Ministère de la Santé et des Agences de coopération. Conakry, Guinée, du 11 au 22 janvier 1998.

Geslin, C., Karki, M., Djibrina, S. Rapport de mission : Evaluation Rapide des Performances des Agents (ERPA), République du Niger, Décembre 1997.

Karki, M., Geslin, C., Kelly, P., Blum, L. Rapport de l'enquête sur la prise en charge de l'enfant malade dans les districts sanitaires de Boboye, Konni et Guidan-Roumji. 12 au 24 mai 1997, République du Niger, Report, BASICS.

Miller $\mathrm{R}$ et al. The Situation Analysis Approach to Assessing Family Planning and Reproductive Health Services : A Handbook Population Council New York, 1997.

Ministère de la Santé. Annuaire des Statistiques Sanitaires 1996. Conakry Guinée. Octobre 1997.

Ministère de la Santé. Normes et Procédures des Services de Santé de la Reproduction. Conakry Guinée. Novembre 1997.

Ministère du Plan et de la Coopération. Bureau National du Recensement. Recensement Général de la Population et de l'Habitation, Conakry Guinée.1996.

Ministère du Plan et de la Coopération. Bureau National du Recensement. Enquête Démographique et de Santé.Conakry Guinée. 1992.

Murray J. et Manoncourt. Integrated Health Facility Assessment Manual : Using Local Planning to Improve the Quality of Child Care at Health Facilities. Arlington BASICS 1997.

Murray, J. \& Manoncourt, S. Manuel d'évaluation intégrée des établissements de santé : La planification locale mise au service d'une meilleure qualité de soins aux enfants dans les établissements de santé. Arlington, BASICS, 1998.

Population Council et Ministère de la Santé du Sénégal. Analyse Situationnelle des Système de Prestation de services de planification Familiale au Sénégal . Dakar Senegal. Octobre 1995 
Population Council et Ministère de la Santé du Sénégal. Evaluation des Programmes de Santé de la Reproduction. Dakar Senegal. Novembre 1997.

Projet d'Appui à la Lutte contre le SIDA en Afrique de l'Ouest/Volet Guinée. Modules de Formation sur le Diagnostique Biologique des MST . Conakry Guinée. Août 1997. 